\title{
Article
}

\section{The consequence of a new ISRF model of the Milky Way on predictions for diffuse gamma-ray emission}

Niederwanger, F., Reimer, O., Kissmann, R., Strong, A.W., Popescu, Cristina and Tuffs, R.

Available at http://clok.uclan.ac.uk/26199/

Niederwanger, F., Reimer, O., Kissmann, R., Strong, A.W., Popescu, Cristina ORCID: 0000-0002-7866-702X and Tuffs, $R$. (2019) The consequence of a new ISRF model of the Milky Way on predictions for diffuse gamma-ray emission. Astroparticle Physics, 107 . pp. 1-14. ISSN 0927-6505

It is advisable to refer to the publisher's version if you intend to cite from the work. http://dx.doi.org/10.1016/j.astropartphys.2018.11.001

For more information about UCLan's research in this area go to http://www.uclan.ac.uk/researchgroups/ and search for < name of research Group>.

For information about Research generally at UCLan please go to http://www.uclan.ac.uk/research/

All outputs in CLoK are protected by Intellectual Property Rights law, including Copyright law. Copyright, IPR and Moral Rights for the works on this site are retained by the individual authors and/or other copyright owners. Terms and conditions for use of this material are defined in the policies page.

\section{CLoK}

Central Lancashire online Knowledge www.clok.uclan.ac.uk

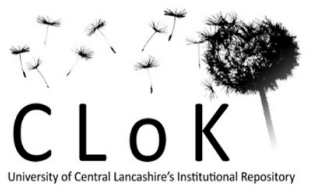




\title{
The consequence of a new ISRF model of the Milky Way on predictions for diffuse gamma-ray emission
}

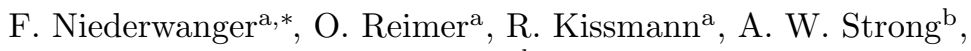 \\ C.C. Popescu ${ }^{\text {c,d }}$, R. Tuffs ${ }^{\mathrm{e}}$ \\ ${ }^{a}$ Institut für Astro- und Teilchenphysik, Leopold-Franzens-Universität Innsbruck, A-6020 \\ Innsbruck, Austria \\ ${ }^{b}$ Max Planck Institut für extraterrestrische Physik, Postfach 1312, D-85741 Garching, Germany \\ ${ }^{c}$ Jeremiah Horrocks Institute, University of Central Lancashire, PR1 2HE, Preston, UK \\ ${ }^{d}$ The Astronomical Institute of the Romanian Academy, Str. Cutitul de Argint 5, Bucharest, \\ Romania \\ ${ }^{e}$ Astrophysics Department, Max Planck Institut für Kernphysik
}

\begin{abstract}
We investigate the impact of the recently published ISRF model of the Milky Way by Popescu et al. on the CR electrons and positrons in the context of cosmic-ray transport modelling. We also study predictions for diffuse Galactic gamma radiation and underline the importance of the increased ISRF intensities. We use the PICARD code for solving the CR transport equation to obtain predictions based on Galactic cosmic-ray electron fluxes. We show that the new ISRF yields gamma-ray intensity increases, most particular at the Galactic Center and in the Galactic Plane. The impact is largest at energies around $220 \mathrm{GeV}$.
\end{abstract}

Keywords: Cosmic Rays: electron transport, Methods: numerical, Gamma-rays: general, high-energy, inverse Compton process

\section{Introduction}

The gamma-ray sky above $100 \mathrm{MeV}$ is dominated by diffuse Galactic emission (DGE) [5], which is produced by cosmic rays (CR) that are interacting with the Galactic gas and the interstellar radiation field (ISRF). The DGE is an important source for background radiation of gamma-ray observations within the Galactic Plane and the Galactic halo. Since cosmic rays are deflected and fragment/decay while they propagate through the Galaxy, diffuse gamma-ray emission provides a suitable tool to study their origin and propagation properties [50].

There is indication for an excess in the diffuse gamma-ray emission above $10 \mathrm{GeV}$ in the Galactic Center and in the Galactic Plane with respect to widely used models [5, 29, 34, 30] based on the GALPROP $\operatorname{cod}^{1}$ [4, 30]. One of the applications are the models of the Fermi-LAT collaboration [4], which have been tuned and/or use re-normalized $\mathrm{CR}$ fluxes in order to reproduce the local observational data and are able to accurately describe a wide number of experiments. Alternative models propose different solutions to the problem, e.g. by introducing galactocentric rigidity dependence of the diffusion coefficient (DRAGON Codf2] 21]). Charged cosmic-rays

\footnotetext{
* Corresponding author

Email address: Felix.Niederwanger@uibk.ac.at (F. Niederwanger)

1 https://galprop.stanford.edu/

2 http://dragonproject.org
} 
can only be directly measured at Earth, and especially high-energy electrons do not propagate far from their sources, thus making it difficult to deduce the CR distribution in the inner Galaxy, where the diffuse emission is expected to reach its maximum [9]. Additionally, different intensities and spectral shapes of the ISRF components consequently affect gamma-ray predictions from regions like the Galactic Center.

Prediction for the ISRFs of the Milky Way have been provided by the commonly used GALPROP model [45, 36, 46, based on models for the stellar emissivity and dust distributions derived from star counts and gas measurements, which are known to have limitations. Recently, a new model of the stellar and dust radiation fields has been released [42, which matches the relevant data from the Milky Way. We show predictions of a numerical CR propagation model for the gamma-ray emission using the new ISRF model.

\section{The interstellar radiation field model of the milky way}

The new ISRF model of the Milky Way 42 was derived using realistic radiative transfer models, that were successfully used to explain the spectral energy distribution (SED) of individual nearby galaxies [39, 40, 41, as well as for the statistical behaviour of large samples (e.g. [15, 17, 18, 19, 27, 28, 37, 38, 51]). When applied to the Milky Way, these radiative transfer models were adapted to the internal view of the Galaxy and to constraints imposed by our edge-on view of the Galaxy from the position of the Sun. The spatial and spectral distribution of the ISRF, together with the stellar and dust distributions were derived in a self-consistent way by iteratively comparing models with observations from COBE, IRAS, and PLANCK. The model is thus consistent with imaging data from the near-infrared to the sub-millimeter regime. The ISRF consists of two components: stellar-, and dust-emission light. We complement this with the cosmic microwave background according to Planck's law [4].

The ISRF model is defined on a cylindrical coordinate system $(r, z)$ at different wavelengths $\lambda: S=S(r, z, \lambda)$. The frequency binning allows for accurate inclusion of prominent lines and has higher resolution than previous models. The new ISRF model comprises 135 frequency bins, with 15 bins representing the starlight and 120 bins the dust-emitted light component. The spatial grid spans 22 points in the $r$ and $z$ direction, respectively ranging from 0 to $24 \mathrm{kpc}$ in radial direction and 0 to $10 \mathrm{kpc}$ in vertical direction. Those points are on a logarithmic scale, leading to a high-resolution description near the Galactic Center. The ISRF intensity decreases notably with increasing distance from the Galactic Center, with intensities changing by nearly two orders of magnitude for the starlight component between the Galactic Center and Earth. Figure 1 shows the starlight and dust emission component of the ISRF model at different distances from the Galactic Center.

The starlight component in the ISRF model in Popescu et al. 42] reaches up to $5 \mu \mathrm{m}$. At this wavelength, the starlight intensity is still approximately an order of magnitude larger than the dust-emitted light. We therefore extend the starlight component to longer wavelengths by using a simple power-law fit on the last two available wavelength bins.

The dust emission component ranges from $3 \mu \mathrm{m}$ to $1 \mathrm{~mm}$. For shorter and longer wavelengths we use extrapolation on the last two available datapoints. There, however, the dust component is already subdominant to the starlight or CMB emission.

\subsection{The ISRF model in PICARD}

We map the new ISRF model onto the equidistant grid used by PICARD (see section 3) via logarithmic interpolation. As an example Figure 2 shows the ISRF 


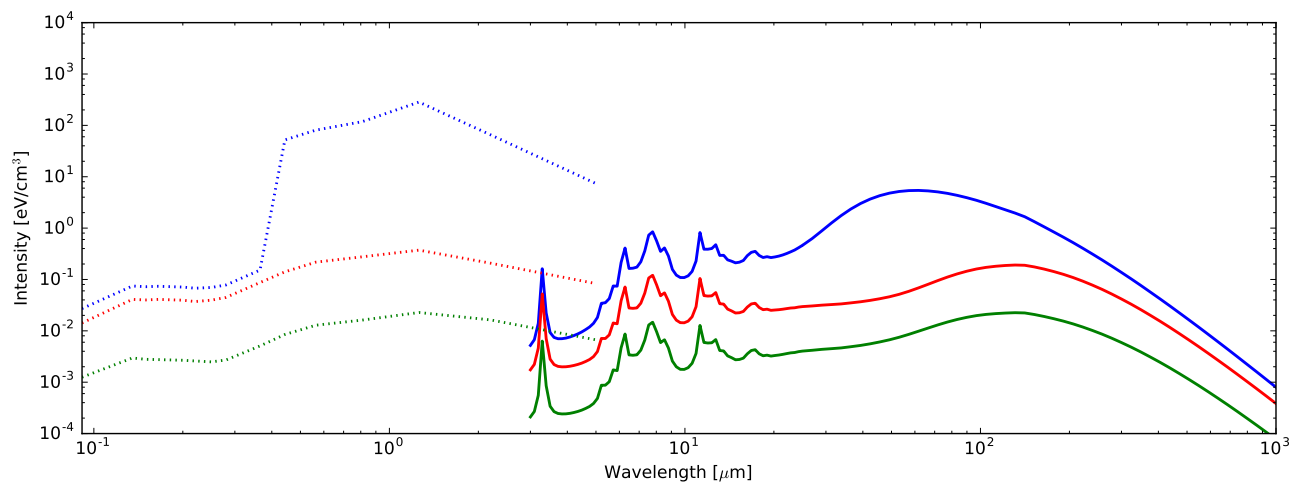

Figure 1: Spectrum of the ISRF components (Starlight is dotted and dust-emitted light is solid) at the Galactic Center (blue), Earth (red) and at $R=15 \mathrm{kpc}, z=0 \mathrm{kpc}$ (green)

model and the mapped ISRF in PICARD at a wavelength of $100 \mu \mathrm{m}$.

Henceforth we refer to the Popescu et al. ISRF model [42] that has been mapped to the PICARD grid as the PICARD ISRF model.

\subsection{Radial and vertical profiles}

Because of its axisymmetric nature, vertical and radial profiles of the ISRF intensity are the most natural way to discuss the spatial dependence of the PICARD ISRF model.

Figure 3 shows radial profiles whereas Figure 4 shows vertical profiles for the GALPROP ISRF model and the PICARD ISRF model at selected wavelengths, respectively.

The intensity gradients, both in the radial and the vertical direction, are different towards the Galactic Center and the Galactic Plane respectively. For the inner $1 \mathrm{kpc}$ the radial profiles show an order-of-magnitude general intensity increase for the starlight- and dust-dominated wavelength regimes and similar intensities towards the CMB regime.

Between $2 \mathrm{kpc}$ and $5 \mathrm{kpc}$ the radial profile is nearly flat. Similar to the radial profile, the vertical profile shows an increase in the inner $0.5 \mathrm{kpc}$ up to $1 \mathrm{kpc}$, depending on the wavelength, and a flatter profile at a height larger than $1 \mathrm{kpc}$. In general, the PICARD ISRF has higher intensities towards the Galactic Plane than the GALPROP ISRF in the dust dominated regime.

\subsection{Spectral comparison with previous model}

Figure 5 shows the spectral energy distribution of both ISRF models at Earth and at the Galactic Center. In contrast to the GALPROP ISRF model, the new ISRF model has three distinct new features: A substantial intensity increase towards the Galactic Center, a generally larger intensity in the dust emission regime, and below $90 \mathrm{~nm}$ an overall reduced intensity in the starlight regime.

The ISRF-intensity increase in the Galactic Center dominates for wavelengths above $0.4 \mu \mathrm{m}$ and is limited to the inner $0.5 \mathrm{kpc}$ of the Galaxy. At $0.5 \mathrm{kpc}$ the intensities of the two ISRF models are comparable for wavelengths between $1 \mu \mathrm{m}$ and $10 \mu \mathrm{m}$.

In the dust-dominated regime, the intensity increase is most obvious between $3 \mu \mathrm{m}$ and $200 \mu \mathrm{m}$. At Earth the effect amounts to between a factor of 1.5 at $3 \mu \mathrm{m}$ and a 


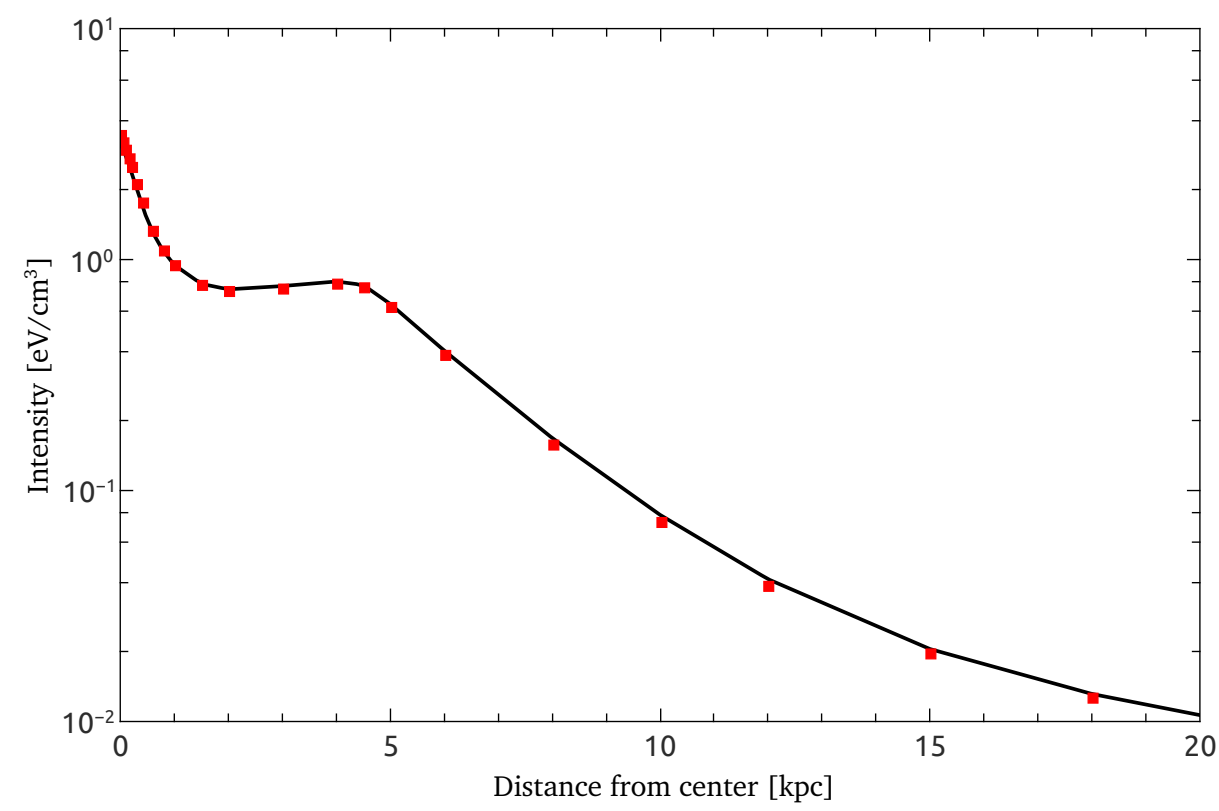

Figure 2: Radial profiles of the Popescu et al. ISRF model (red dots) and corresponding intensity mapped onto the equidistant PICARD grid (black solid line) at $100 \mu \mathrm{m}$.

factor of 3 at $30 \mu \mathrm{m}$. At longer wavelengths $(>200 \mu \mathrm{m})$ the two ISRF models have comparable intensities.

In the starlight regime, below $90 \mu \mathrm{m}$ for Earth and $2 \mu \mathrm{m}$ for the Galactic Center, the intensity of the new ISRF model is smaller compared to the GALPROP ISRF model.

Apart from this, the spectral resolution of the new ISRF is larger, especially in the dust dominated regime. For example the diffuse interstellar band features $10 \mu \mathrm{m}$ are captured more clearly than in previous models used in CR propagation codes with practical no visible consequences for the gamma-ray emission (See section 3). Reducing the spectral resolution to the one used by GALPROP does not lead to any obvious changes for the gamma-ray emission. Both ISRF models converge with increasing wavelength and are identical in the CMB.

Here we note that the amplification of the NIR radiation fields at the Galactic Center in [42] strongly depends on the assumed optical constants of the dust, which were taken to be the canonical values from [52. These dust absorptivies and emissivities, although empirically anchored at laboratory measurements in the UV/optical/NIR/FIR range, are quiet uncertain in the submm regime. If the submm grain emission efficiencies were higher than predictions of the WD01 model [52, this would be an overestimation of the UV-optical depth of the Galaxy.Since the amplitude and spectrum of the stellar emission in the NIR was derived in 42 by fitting the NIR imaging of the directly observed stellar light, the radiation fields depend sensitively on the assumed dust properties. However, the NIR radiation fields are not important contributors to the diffuse gamma-ray emission. On the other hand, the FIR/submm ISRFs, which are the dominant source of seed photons for the IC emission, are highly robustly determined, since they are directly mapped into the FIR emissivities.

Apart from the NIR regime, the Popescu et al. 42 model for the UV/optical and 

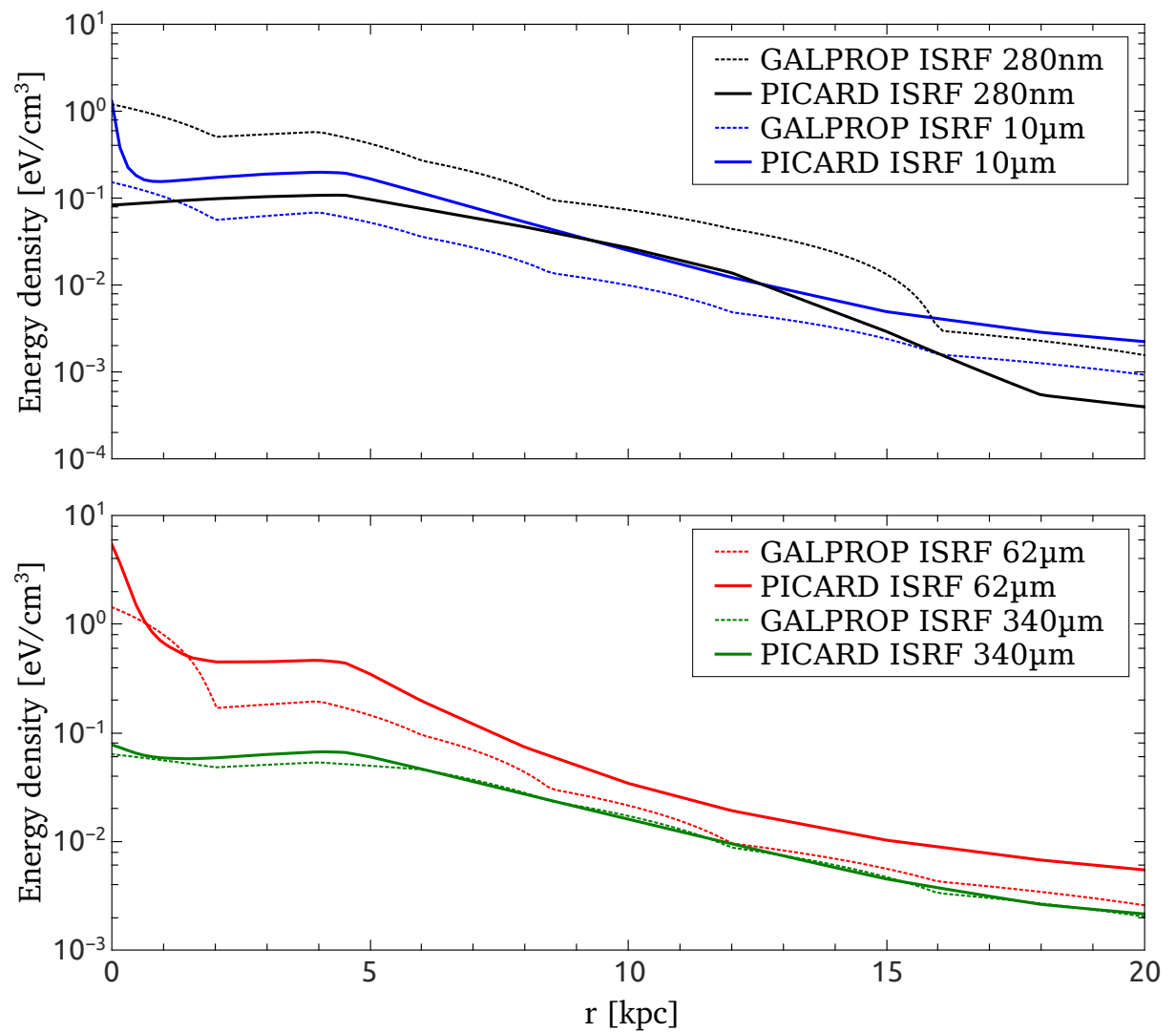

Figure 3: Radial profiles at $z=0 \mathrm{kpc}$ of the two ISRF models investigated in this study at different wavelengths. Solid is the PICARD ISRF model, dotted the GALPROP ISRF model. 

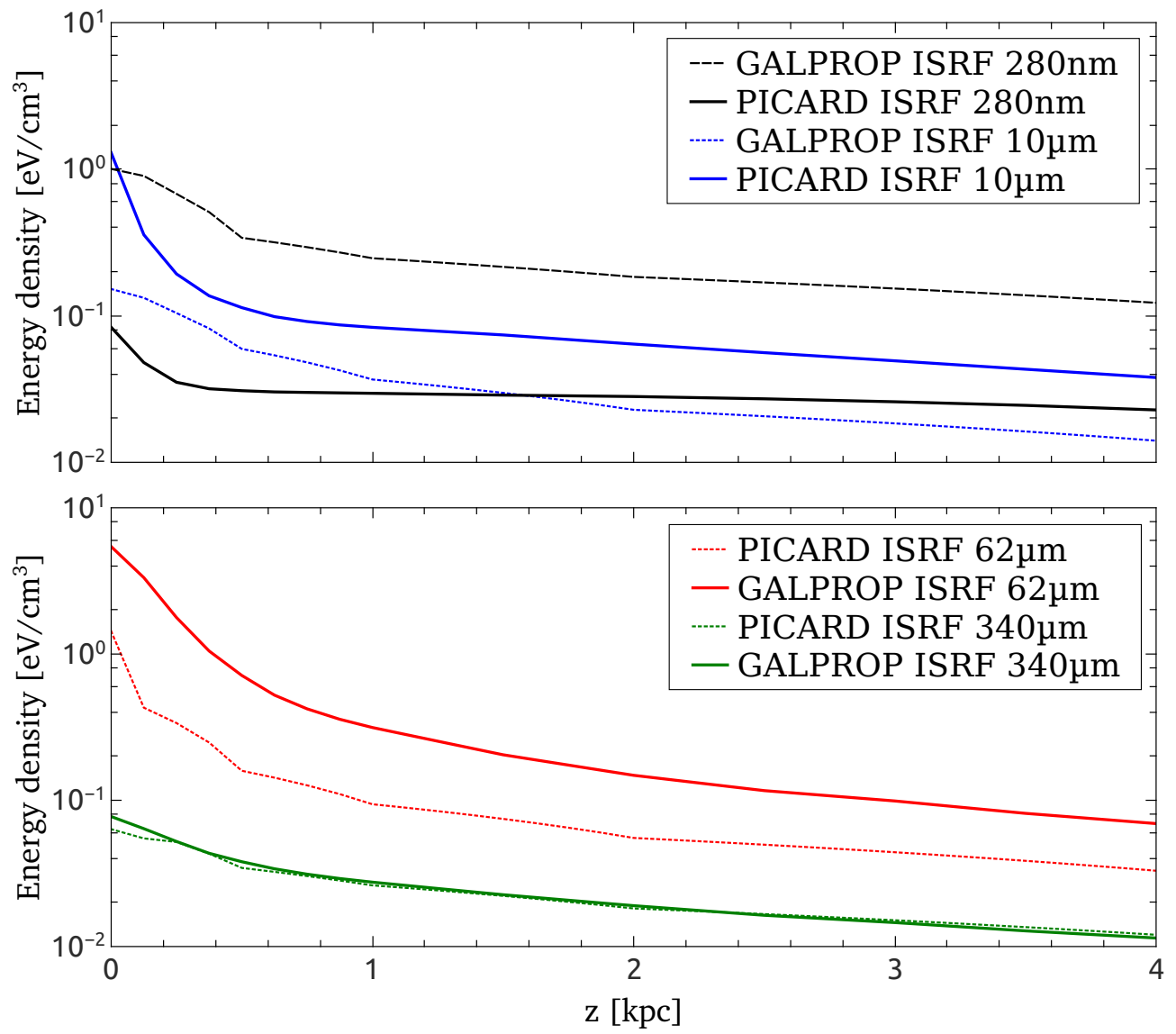

Figure 4: Same as Fig. 3, but for the vertical profiles.

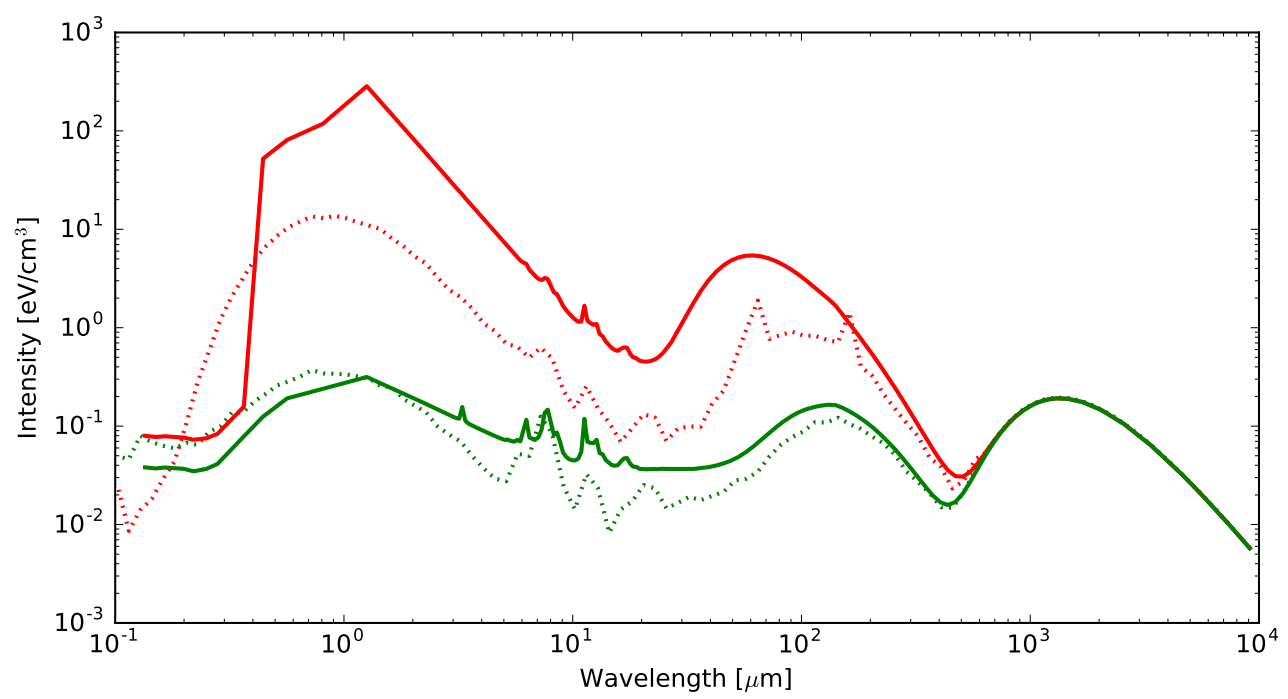

Figure 5: SED for the two ISRF models at the Galactic Center (red, upper) and Earth (green, lower), respectively. Solid line for PICARD ISRF, dotted line for GALPROP ISRF. 
MIR/FIR/submm energy densities is invariant to the choice of the dust model.

\section{Cosmic-ray propagation model}

We use the PICARD code 47, for numerical modelling of the CR propagation. Our CR propagation model cites the propagation parameters ${ }^{\mathrm{S}} \mathrm{Y}^{\mathrm{z}} 4^{\mathrm{R}} 20^{\mathrm{T}} 150^{\mathrm{C}} 5^{3}$ from [5] using an axisymmetric CR source distribution based on pulsars from [54. The electron injection spectrum is a broken power law with spectral index of $1.6(\leq \mathrm{GeV})$, $2.42(\sim 2 \mathrm{GeV}$ to $2 \mathrm{TeV})$ and $4(>2 \mathrm{TeV})$.

We compare with the publicly available GALPROP code v54 and the thereby provided ISRF model referred further on as GALPROP ISRF model [4, 45].

We perform simulations for both ISRF models using $257 \times 257 \times 65$ grid points in space, covering an extent from -20 to $20 \mathrm{kpc}$ in $x$ and $y$ direction and -4 to $4 \mathrm{kpc}$ in $z$ direction, and 127 points in momentum space ranging from $10 \mathrm{MeV}$ to $1 \mathrm{PeV}[33$ ]

Predictions of the gamma-ray emissivity include Bremsstrahlung, neutral pion decay, and IC emission for an energy range between $50 \mathrm{MeV}$ and $10 \mathrm{TeV}$.

\section{Application of the ISRF}

In this section we investigate the effect of the new ISRF on cosmic ray propagation (Sections 4.1 and 4.2 ) and the subsequent prediction of gamma-ray emission (Section 4.3 , based on the transport model discussed in 3

\subsection{Energy loss rates}

In the following we consider Bremsstrahlung, Coulomb scattering, ionisation losses, IC and synchrotron radiation. Figure 6 shows the individual and total energy loss rates for electrons at the Galactic Center. The order-of-magnitude intensity increase in the PICARD ISRF compared to the GALPROP ISRF translates to a considerable increase of IC losses up to roughly $100 \mathrm{TeV}$. At higher energies the Klein-Nishina cross section equalizes the IC loss rate between the two ISRF models, with synchrotron already being the dominant loss process. IC losses dominate the total energy loss rate, up to $\sim 300 \mathrm{GeV}$ for the GALPROP ISRF model or up to $1.5 \mathrm{TeV}$ for the PICARD ISRF model.

As discussed in [42], the most relevant seed photons for IC gamma-rays in the $\mathrm{GeV}$ to $\mathrm{TeV}$ regime originate from the dust-dominated regime because higher energy seed photons from the starlight regime are already in the relativistic Klein-Nishina regime.

\subsection{Cosmic-ray fluxes}

Figure 7 shows results from modelling electron and positron fluxes at the Galactic Center and at Earth using the two different ISRF models. The electron spectrum fits a simple power-law from $10 \mathrm{GeV}$ to $500 \mathrm{GeV}$ and above $3 \mathrm{TeV}$, with the breaks stemming from the electron injection spectrum. The electron and positron spectra at Earth are very similar for both ISRF models. The spectral index of the electron flux for the energy range between 10 and $500 \mathrm{GeV}$ is $\approx 3.08$ for the GALPROP ISRF and $\approx 3.10$ for the PICARD ISRF model. For comparison, PAMELA data indicate a spectral index of $3.18 \pm 0.05$ above $30 \mathrm{GeV}$ [7, Fermi-LAT of 3.04 between $20 \mathrm{GeV}$

\footnotetext{
${ }^{3}$ Nomenclature taken from [5], The radial extent is $R=20 \mathrm{kpc}$, the height $z=4 \mathrm{kpc}, T_{S}=150 \mathrm{~K}$ is a assumed unified hydrogen spin temperature, and the $E(B-V)$ cut $=5 \cdot 10^{20} \mathrm{~cm}^{-2} \mathrm{mag}^{-1}$.
} 

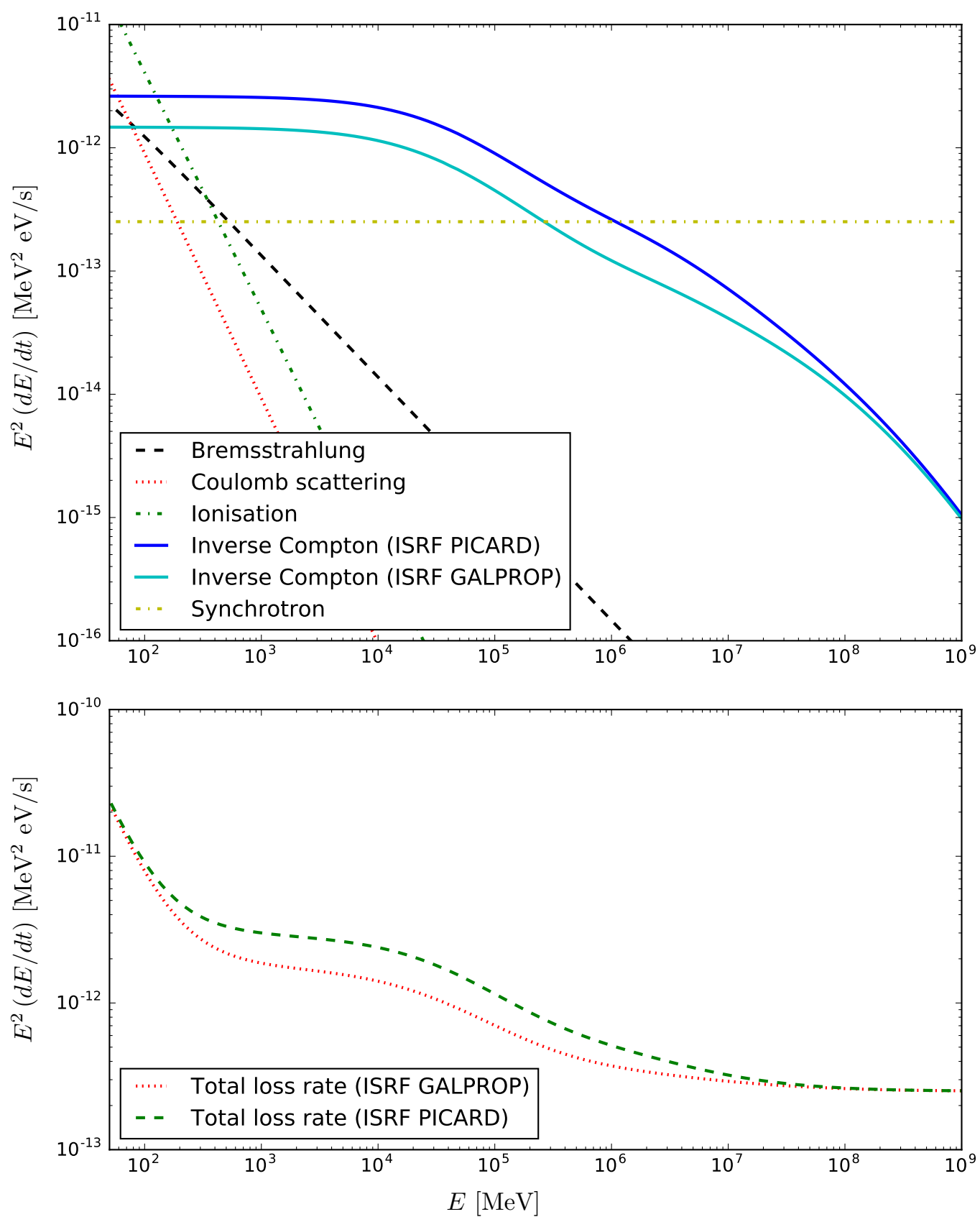

Figure 6: Individual (upper panel) and total (lower panel) energy loss rates for electron at the Galactic Center for both ISRF models. 

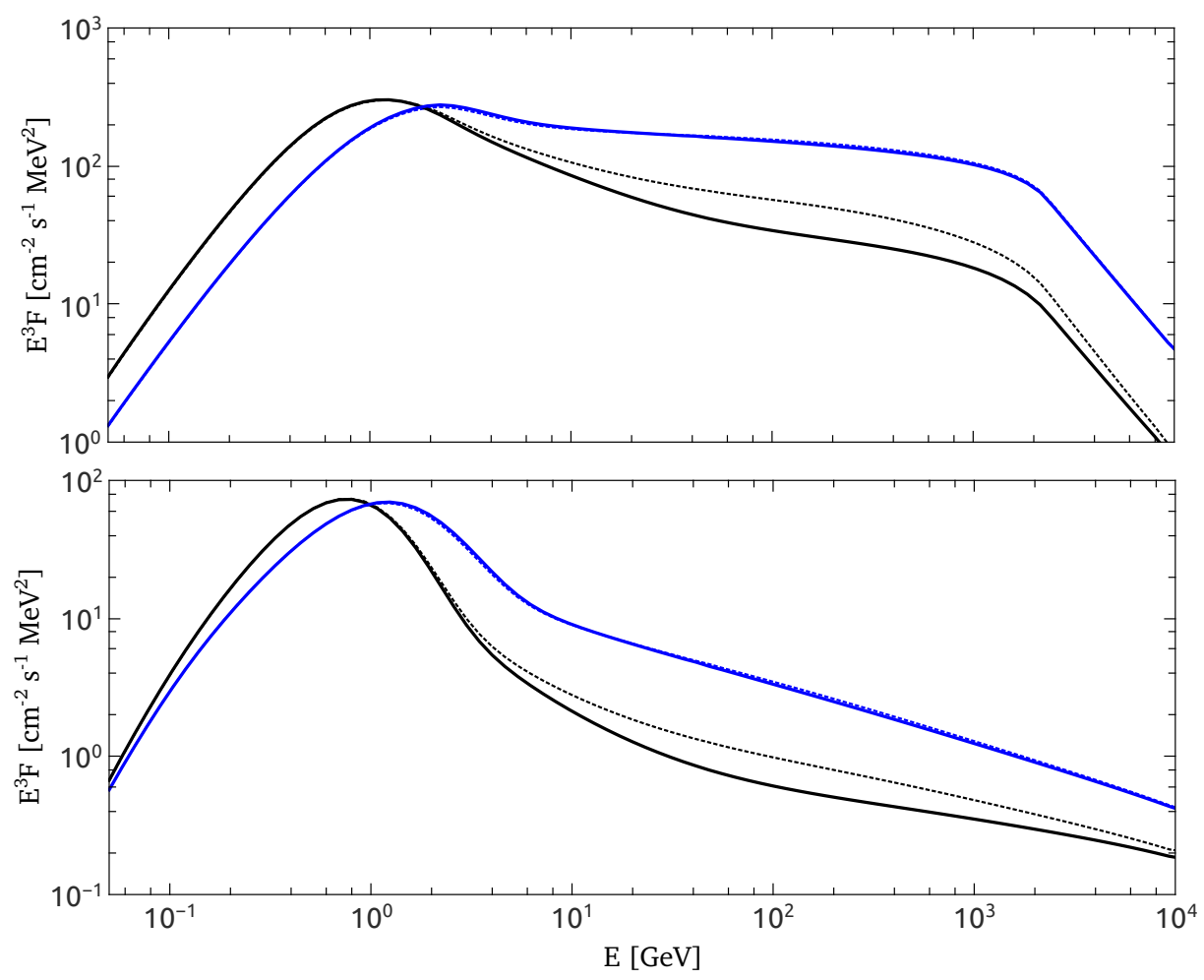

Figure 7: Upper panel: Electron spectra at Earth (blue) and the Galactic Center (black) for the GALPROP ISRF model (dotted) and the PICARD ISRF model (solid), lower panel: likewise for positrons

and $1 \mathrm{TeV}[2]$ and H.E.S.S. data an index of $3.05 \pm 0.02$ with a softening at $2.1 \pm 0.3$ $\mathrm{TeV}$ 8. DAMPE reports a spectral index of 3.1 for electrons above $>55 \mathrm{GeV}$, with a spectral break at $\approx 0.9 \mathrm{TeV}$ to a spectral index of 3.9 up to $2.63 \mathrm{TeV}[10]$ and CALET reports a single electron power law in the range from $10 \mathrm{GeV}$ to $3 \mathrm{TeV}$ with a spectral index of $-3.152 \pm 0.0166$.

In our models, at higher energies, the electron spectrum softens to $4.70 \pm 0.05$ between $3 \mathrm{TeV}$ and $10 \mathrm{TeV}$ for both ISRF models.

In the Galactic Center, the use of the new ISRF leads to a considerable softening of the electron spectrum above $3 \mathrm{GeV}$. The corresponding decrease in the electron flux between $3 \mathrm{GeV}$ to $10 \mathrm{TeV}$ is at most $40 \%$ at $150 \mathrm{GeV}$.

Given the underrepresentation of positrons against electrons and the used assumption of the same transport physics, positrons will only contribute marginally to the diffuse gamma-ray emission.

\subsection{Gamma-ray emission}

We distinguish between the local gamma-ray emissivity and their line-of-sight integrals, the diffuse gamma-ray emission (DGE).

\subsubsection{Local gamma-ray emissivity}

The spatial and spectral differences in the two ISRF models lead to different local IC emissivities, i.e. the observed higher energy loss rates in the Galactic Center (see 


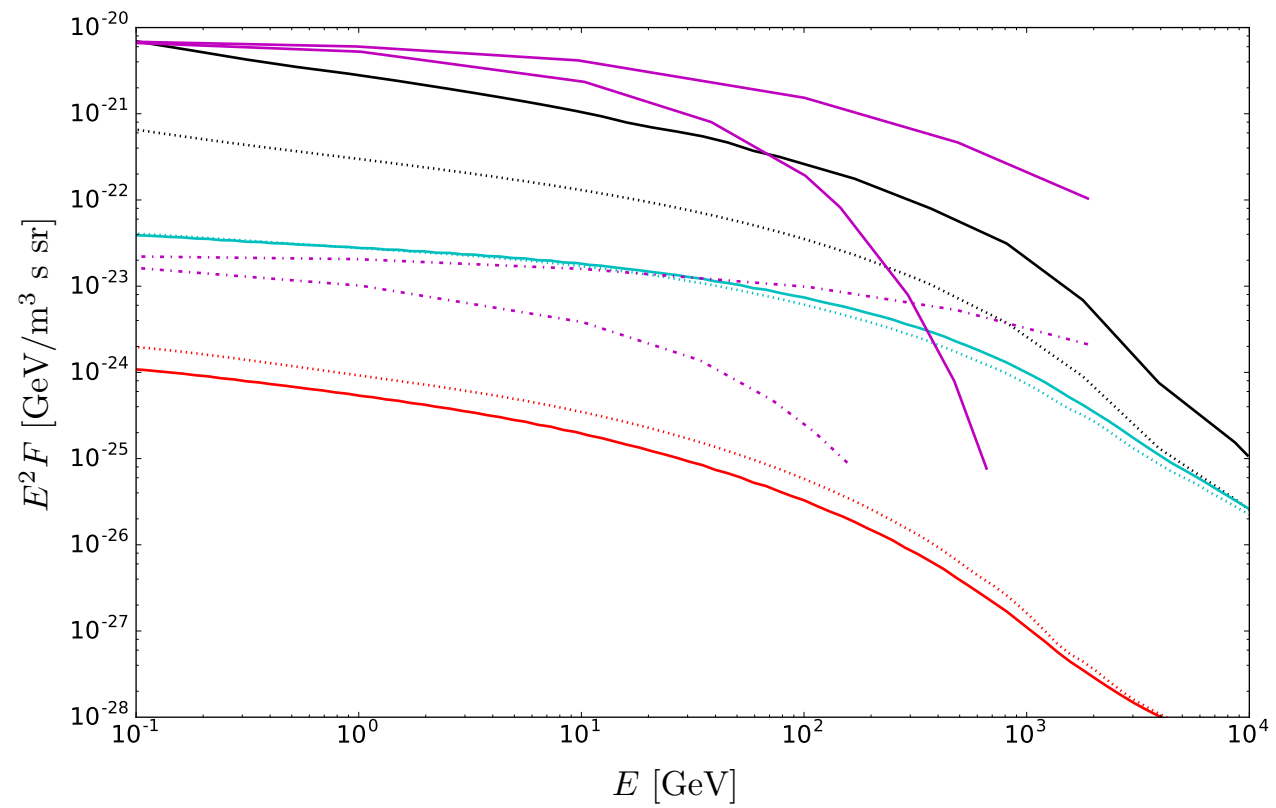

Figure 8: Local IC gamma-ray emissivities for both ISRF models in the Galactic Center (black), at Earth (cyan) and at a halo position $((10,10,2) \mathrm{kpc}$, red). Solid lines are for the emissivities using the PICARD ISRF model and dotted lines for using the GALPROP ISRF model. Magenta and green shows the IC gamma-ray emissivities from [42, Figure 13] for comparison - Green shows the Galactic Center, Magenta Earth with electron-cut-off energies $E_{b}=0.01 \mathrm{TeV}$ (dashed) and $E_{b}=1 \mathrm{TeV}$ (dash-dotted). 


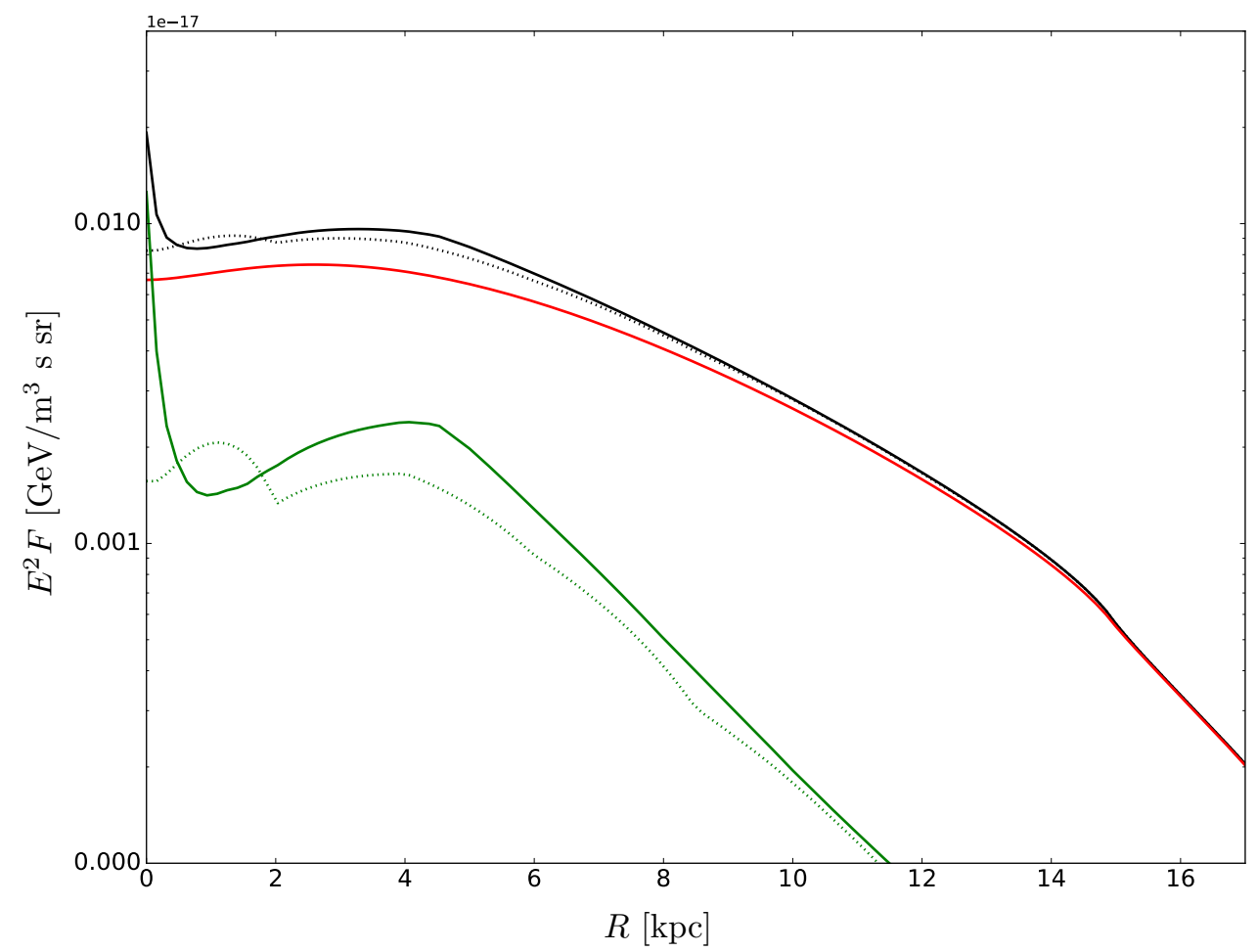

Figure 9: Radial profile of the IC (green) and total (black) gamma-rays emissivities at $220 \mathrm{GeV}$ for both ISRF models. Results using the PICARD ISRF model are shown as solid lines whereas those for the GALPROP ISRF model are shown in dotted lines. Neutral pion decay emissivities are shown for comparison only (solid red).

section 4.1) are also reflected as correspondingly higher IC emissivities there, despite the significant reduction of the electron flux. Figure 8 shows the spectrum for the IC-related gamma-ray emissivity at the Galactic Center, Earth and a halo position at $[10,10,2] \mathrm{kpc}$ for our models and for comparison also the IC emissivities from [42 Figure 13]. The spectra following from the different ISRF models are similar in shape, but the model using the new ISRF leads to higher emissivities in the Galactic Center by up to an order of magnitude. At Earth the IC emissivities are comparable and at the halo position the new ISRF model decreases the IC emissivity by approximately a factor of two.

Figure 9 then shows radial profiles for the IC, the neutral pion decay, and the total gamma-ray emissivities at $220 \mathrm{GeV}$.

There is a notable increase in IC emission towards the Galactic Center, where IC becomes the dominant gamma-ray emission channel. Between $100 \mathrm{GeV}$ and $1 \mathrm{TeV}$ this characteristics is seen in a 150 pc-scale region around the Galactic Center, reaching the present spatial resolution limit in our simulations.

This IC emissivity increase in sub-kpc scales motivates further studies at higher resolution (see section 5).

In contrast to [42, where a spatially constant CR electron spectrum has been assumed, our results show notably different energy dependencies of the gamma-ray emissivities at different galactocentric radii.

The highest gamma-ray emissivities outside of the Galactic Center are found at a 


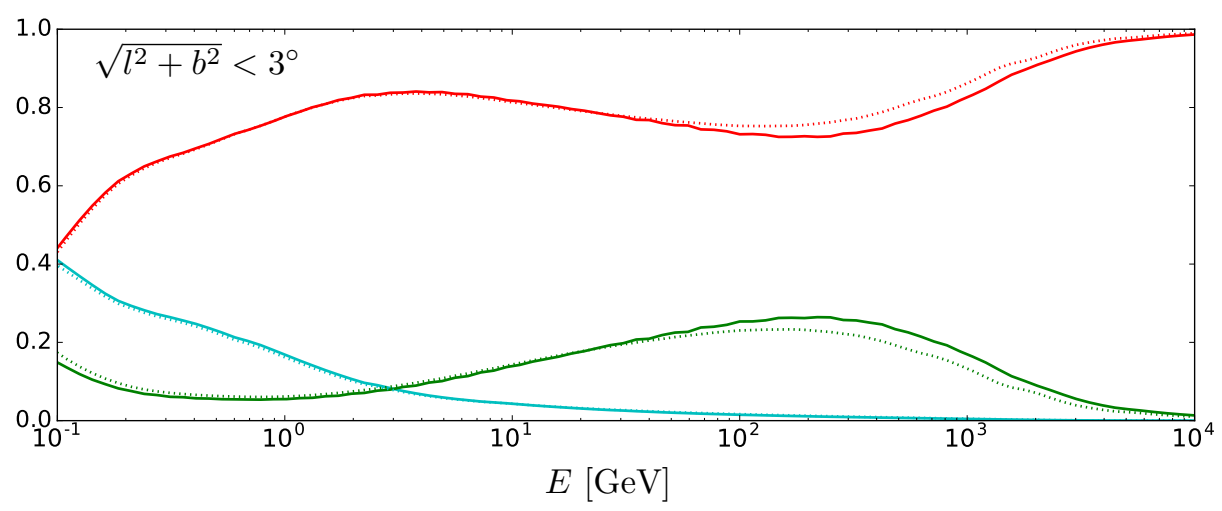

Figure 10: Relative contributions of the different gamma-ray production channels to the DGE for the Galactic Center region. Neutral pion decay emission is in red, IC in green, Bremsstrahlung cyan. Dotted line for GALPROP ISRF model, solid lines for the PICARD ISRF model.

distance of $\sim 5 \mathrm{kpc}$ from the Galactic Center, coinciding with the peak in the chosen cosmic-ray source-distribution [54].

Increased IC emissivity with respect to the GALPROP ISRF model are found between $2 \mathrm{kpc}$ up to approximately $10 \mathrm{kpc}$ for gamma-ray energies higher than $10 \mathrm{GeV}$.In particular, the total emissivity increases considerably between $3 \mathrm{kpc}$ and $6 \mathrm{kpc}$ and energies above $100 \mathrm{GeV}$ for the PICARD ISRF model. The increase is a net effect of the used CR source distribution and the increased ISRF intensities. A net increase towards the Galactic Center (for the inner $0.1 \mathrm{kpc}$ to $0.3 \mathrm{kpc}$ depending on the gamma-ray energy) is visible at all energies.

\subsubsection{Relative contributions to the DGE}

In this section we analyse the contributions of the individual radiative processes to the line-of-sight integrated total DGE. The contributions are studied in the following directions: Galactic Center $\left(\sqrt{l^{2}+b^{2}}<3^{\circ}\right)$, Galactic Plane $\left(|b| \leq 5^{\circ},|l| \leq 180^{\circ}\right)$, intermediate latitudes $\left(30^{\circ}<|b|<90^{\circ}\right)$, and the outer Galaxy $\left(|b|>8^{\circ}\right.$ or $\left.|l|>100^{\circ}\right)$.

Figure 10 shows the contributions of the individual gamma-ray production channels for the Galactic Center region. This is representative for all studied regions.Neutral pion decay is the dominant gamma-ray production channel in all cases, with considerable contribution of the IC channel to the total gamma-ray emission at energies around $200 \mathrm{GeV}$. The PICARD ISRF model increases the IC gamma-ray flux considerably between $100 \mathrm{GeV}$ and $10 \mathrm{TeV}$.IC related gamma-ray flux is maximum at $220 \mathrm{GeV}$ for the Galactic Center region and the Galactic Plane.

At this energy IC contributes about $26 \%$ towards the Galactic Center, $25 \%$ in the Galactic Plane, and $32 \%$ at intermediate latitudes and the outer Galaxy to the total gamma-ray flux. Lower IC losses and corresponding lower gamma-ray fluxes at higher energies relate to the transition into the Klein-Nishina-Regime.

\subsubsection{Gamma-ray spectra}

Figure 11 shows the gamma-ray emission spectra for the three gamma-ray production channels (neutral pion decay, Bremsstrahlung, IC) for the two ISRF models at the regions described in section 4.3.2. The DGE spectra of the two different ISRF models generally agree with each other. Owing to the increased IC emission related to the new ISRF, also the total DGE increases between $20 \mathrm{GeV}$ and $10 \mathrm{TeV}$ by up to $5 \%$. The slight decrease in IC emissivity between $1 \mathrm{GeV}$ and $10 \mathrm{GeV}$ translates to a negligible decrease of the total DGE of less than $1 \%$. The impact of the new 


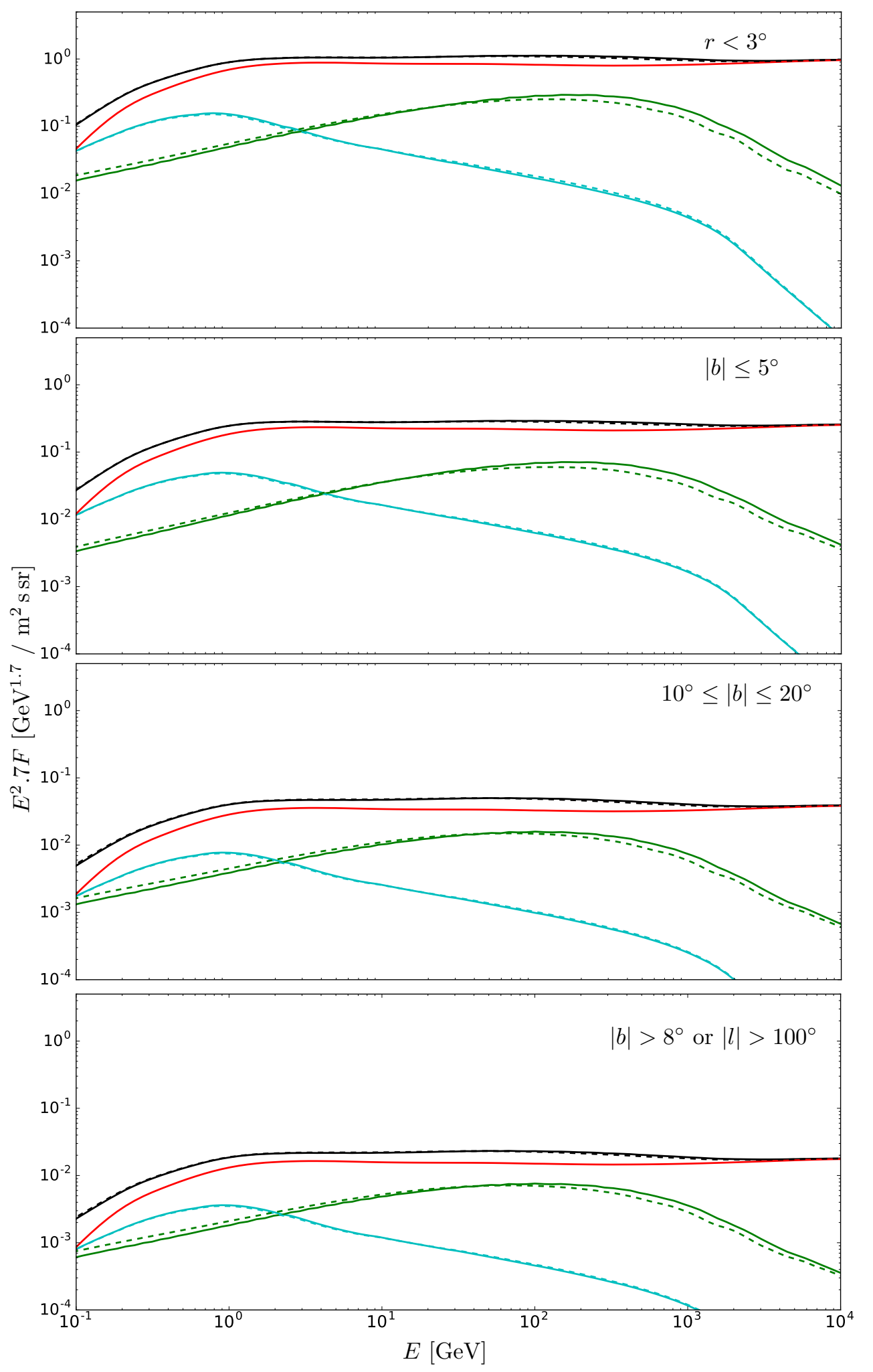

Figure 11: Gamma-ray emission spectra for different regions on the Sky. From top to bottom results are shown for: the Galactic Center, the Galactic Plane, intermediate latitudes, and the outer Galaxy. The specific extent of the regions is given in the respective sub-figure. Note that we use the same extent for the $y$-axis in all plots. Black is for total DGE, red is for $\pi^{0}$-decay, green for IC and cyan for Bremsstrahlung. Solid line for the (PICARD ISRF), dashed line for the (GALPROP ISRF). 
ISRF on the gamma-ray emission differs for the different regions, but is highest for the Galactic Center and the Galactic Plane.

The PICARD ISRF model increases the spectral index with respect to the GALPROP ISRF model on average by 0.01 between $500 \mathrm{GeV}$ and $10 \mathrm{TeV}$ for all regions. Between $15 \mathrm{GeV}$ and $500 \mathrm{GeV}$ the spectrum hardens by 0.01 for the Galactic Center and the Galactic Plane. At intermediate latitudes and the outer Galaxy the spectral hardening is on average 0.02 for the same energy regime. The new ISRF model has a rather small impact on the spectral index on large scales.

\subsubsection{Gamma-ray spatial profiles}

We investigate the longitude and latitude profiles and corresponding residuals for the DGE at $220 \mathrm{GeV}$ where the new ISRF has the largest influence on the gamma-ray emission (See Figure 10). Latitude profiles are shown for $|l| \leq 10^{\circ}$ in Figure 12 and longitude profiles for $|b| \leq 5^{\circ}$ respectively Figure 13 .

The latitude and longitude profiles for both ISRF models agree well with each other but the DGE for the PICARD ISRF model shows an increase in intensity at those energies. A notable increase in diffuse Galactic emission towards the Galactic Center is evident in both spatial profiles. The maximum DGE increases up to $4 \%$ for the latitude profile at $220 \mathrm{GeV}$.Considering the present resolution of the computations near the Galactic Center, it is expected that this difference becomes more pronounced at smaller scales.

In the longitude profile, the two maxima in the residual around $\pm 25^{\circ}$ are the consequence of the IC emissivity increase in the region between $3 \mathrm{kpc}$ and $6 \mathrm{kpc}$ from the Galactic Center (see Figure 9). The central peak in the residual reflect the higher IC emissivities at the innermost few hundred parsecs of the Galaxy.

Figure 14 shows the relative changes of the longitude profile $\left(|b|<5^{\circ}\right)$ for the IC and the total DGE fluxes between the two ISRF models. The IC emission is increased by $25 \%$ at $\pm 30^{\circ}$ from the Galactic Center. The emissivity increase for the inner 0.1$0.3 \mathrm{kpc}$ of the Galactic Center relate to an increase of up to $16 \%$ in the IC flux of the longitude profile. Now for the total DGE, these increases account for changes of $2 \%-5 \%$ in the total DGE profile, being especially interesting for the regions from $-90^{\circ}$ to $60^{\circ}$.

\subsubsection{Gamma-ray skymaps}

In order to illustrate differences between gamma predictions in both ISRFs Figure 15 shows residual maps of the IC and the total DGE emission at $220 \mathrm{GeV}$, respectively. The predictions differe distinctively in three different regions: between $-45^{\circ}$ and $-10^{\circ}$ in longitude, between $10^{\circ}$ and $45^{\circ}$ in longitude, and in the Galactic Center for $r \leq 2^{\circ}$. The former two are consequence of the chosen CR source distribution, and compares well with the longitude profile in Figure 13 At $l= \pm 30^{\circ}$ these structures extent to up to $8^{\circ}$ in latitude. Evidently, the PICARD ISRF model further amplifies features of the underlying axisymmetric source model.

\section{Discussion}

In this paper we have studied effects resulting from the use of the new ISRF model [42] on the diffuse gamma-ray emission in a regime from $\mathrm{GeV}$ to $10 \mathrm{TeV}$. Compared to the widely used GALPROP ISRF model, the PICARD ISRF model introduces three notable changes with consequences for predictions of diffuse gamma-ray emission:

- Order-of-magnitude increased intensity towards the Galactic Center 

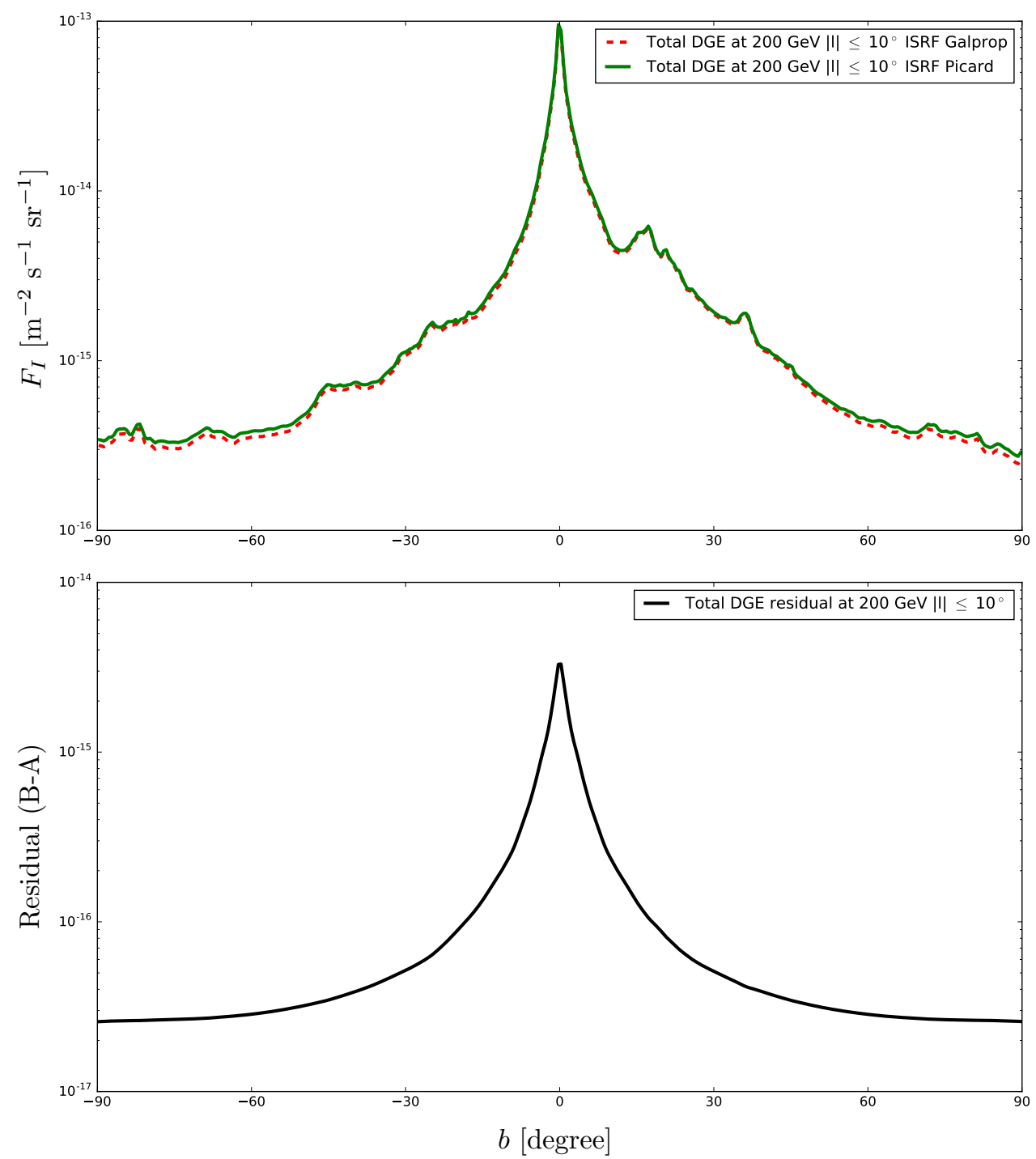

Figure 12: Latitude profile of the total DGE fluxes for $220 \mathrm{GeV}$ from $-10^{\circ}$ to $+10^{\circ}$ longitude for the two ISRF models (top) and residual between the total DGE fluxes (bottom). For the top Figure: Green solid line for the PICARD ISRF model, red dashed for the GALPROP ISRF model. For the residuals: A is the GALPROP ISRF model, B the PICARD ISRF model. 

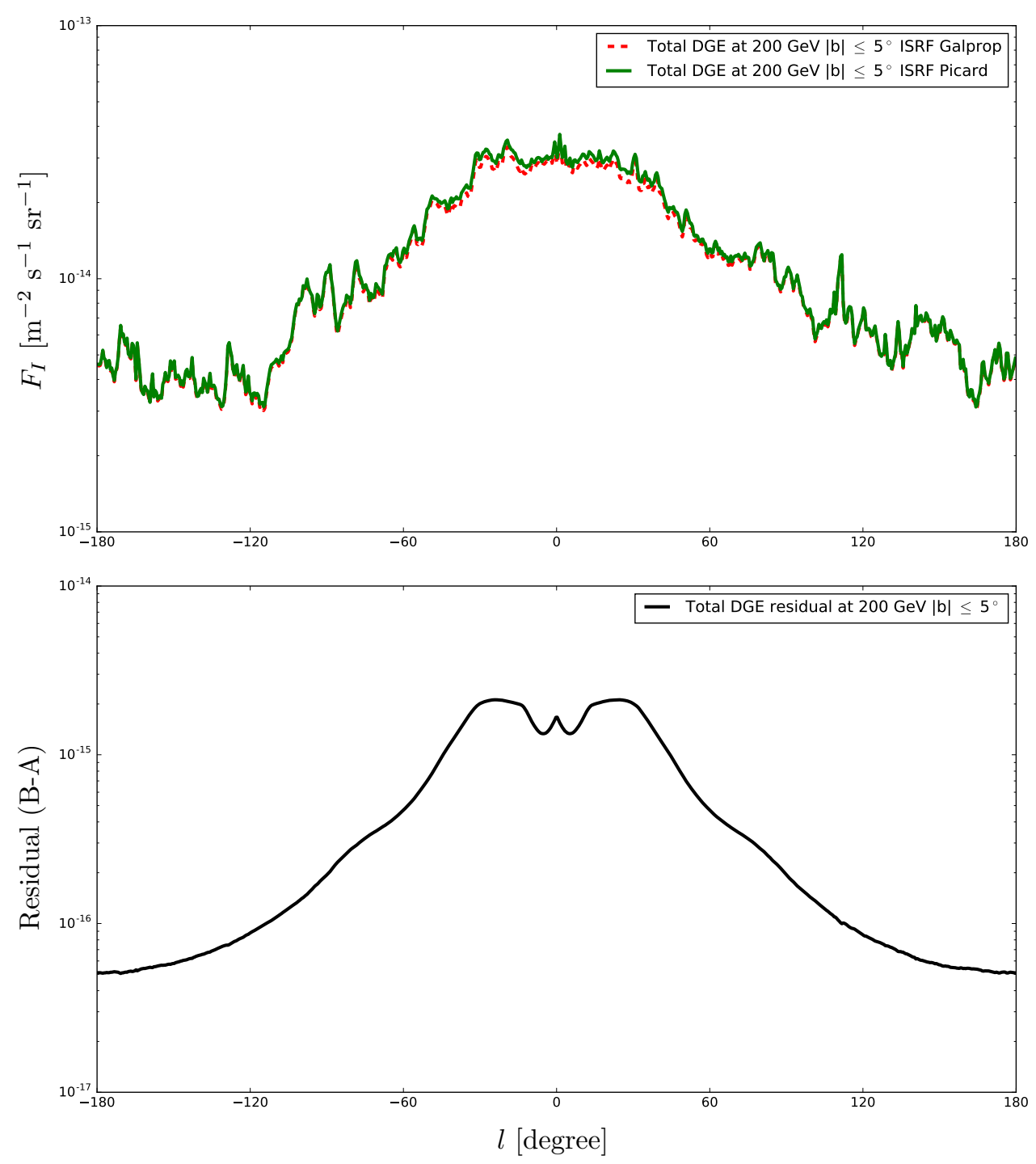

Figure 13: Same as Figure 12 but for the longitude profile over $|b| \leq 5^{\circ}$ 


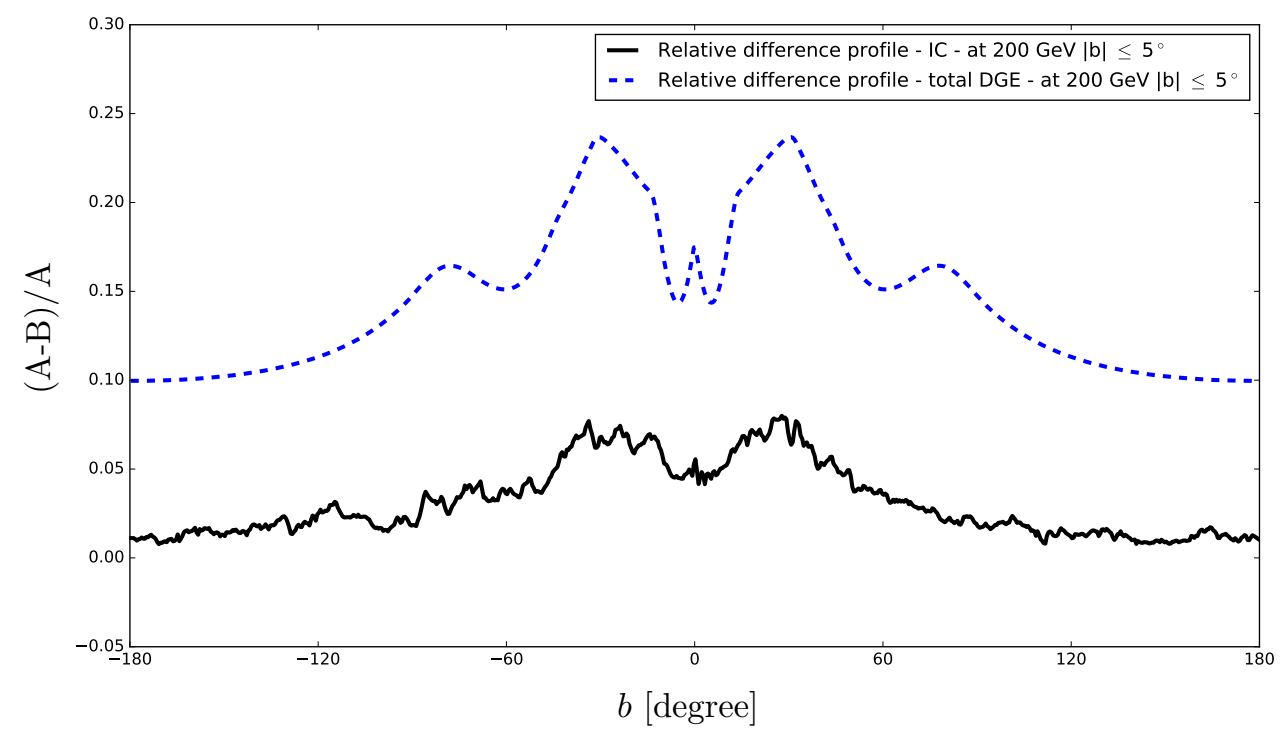

Figure 14: Longitude profile for the relative differences (A-B)/A in the total DGE fluxes (black, solid) and IC fluxes (blue, dashed) at $220 \mathrm{GeV}$ integrated over $-5^{\circ}$ to $+5^{\circ}$ latitude for the two ISRF models.

- Generally increased intensity throughout the dust-dominated regime

- Reduced intensity at shorter wavelengths in the starlight regime

We confirm the findings of Popescu et al. [42] in a cosmic-ray propagation model where we solve the transport equation for an axisymmetric CR source model and show quantitatively the resulting consequences for the DGE.

The local gamma-ray emissivities in the Milky-Way show a considerable increase for all energies in the Galactic Center, that rapidly decreases with increasing Galactocentric distance. For the inner 0.1-0.3 kpc we find an order of magnitude increase in IC emissivity (the size of this region decreases with gamma-ray energy). Thus, at Galactic Center IC becomes the dominant production channel for diffuse gamma-ray emission. Those findings motivate further studies on small scales where the localized emissivity increases are expected to become even more pronounced when using sub-kpc resolution in conjunction with the PICARD ISRF model.

The emissivities at Earth are comparable while showing a mild decrease in the Galactic halo.

Despite a reduction in the electron flux due to the higher intensity of the PICARD ISRF at the Galactic Center, the IC emissivity is increased by an order-of-magnitude. In contrast, higher ISRF intensity in the dust-dominated regime does not affect the energy loss rates and electron spectra at Earth (see Figure 7).

Only recently, the GALPROP ISRF has been revised [44, also leading to increased gamma-ray emission. Likewise, consideration of 3D structure in our Milky-way is advocated regarding the construction of the ISRF [44, the gas distribution 32, and principal execution of galactic propagation [43] as has already been extensively discussed in earlier works using the dynamic Milky-way model 11], DRAGON [22, and PICARD [53, 33. In light of these recent developments we consider it desirable to compare how this alters results and conclusions from earlier numerical models of 

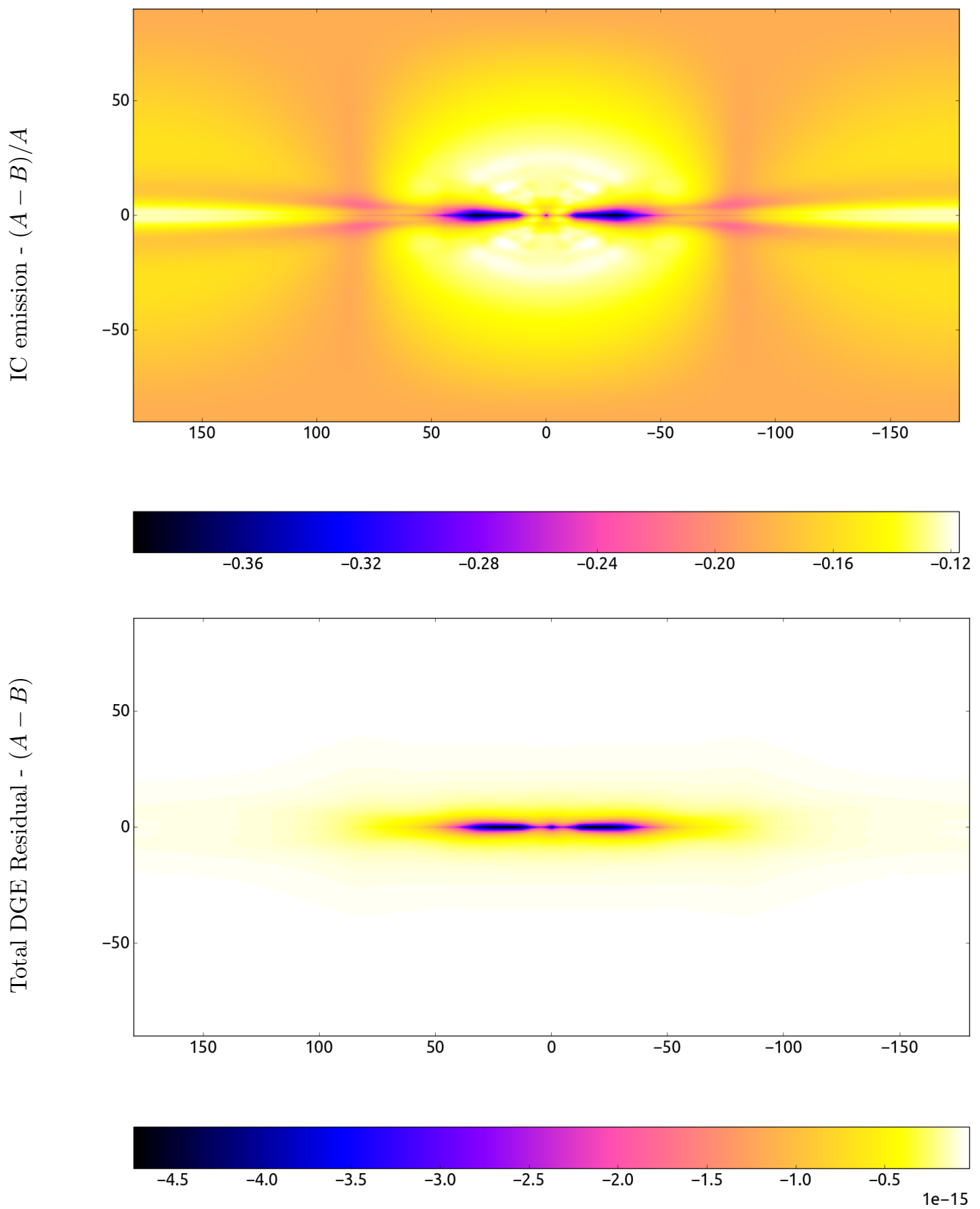

Figure 15: Relative differences in the IC emission (top) at $220 \mathrm{GeV}$ and residual for the total DGE emission (bottom) between the two ISRF models. Positive values indicate a surplus in gamma-ray emission for the GALPROP ISRF model, negative values for the PICARD ISRF model. We use an unbalanced colormap to increase the contrast. 
cosmic ray propagation and associated gamma-ray emission, most particular with emphasis on the IC-induced gamma-ray emission. As the importance of the transition from axisymmetric models for CR source distribution to more realistic models, e.g. including spiral-arms, has been already discussed previously [53, 22, 11] we can compare now realisations of different ISRFs and gamma-ray predictions originating therefrom. Whereas the PICARD ISRF [42] was build upon axial-symmetry, it is constructed on logarithmic binning, allowing for very high resolution towards the Galactic Center: the ISRF portrayed in 44 rests on a 3D-Cartesian Grid with lower resolution in the Galactic Center/Bulge/Disk. This becomes relevant for the intensity increase of the gamma-ray emissivity in the inner 300 pc of our Galaxy, corresponding to the inner $2^{\circ}$, well located in the region, where a noticeable Galactic Center excess has been reported by Fermi-LAT [5]. By using our rather coarse Galaxy model we can only account for a flux increase up to $5 \%$ at the Galactic Center, thus the ISRF model alone lacks an amplification effect that is sufficient large to account for the inferred gamma-ray intensity increase towards the Galactic Center. The ISRF model consequently needs to be discussed in conjunction with more accurate prescriptions for the used CR source component, as e.g. discussed in 53,44 .

Given that observed gamma-ray excess emission towards the Galactic Center [5] is frequently discussed in relation to dark matter in the inner Galaxy [25, 29, 31, 16, 1, 12, 26, the application of the PICARD ISRF [42] in numerical frameworks for Galactic CR propagation, exercised here with PICARD, offers an interesting alternative alongside proposed alterations of the source distribution [14, 30, or different prescriptions of Galactic diffusion in a propagation model like DRAGON $([24,20]$ in explaining the Galactic Center excess. Also the effect of recent injections of high-energy cosmic rays in the Galactic Center region as e.g. suggested by [13, 14 would additionally enhance the respective gamma-ray yields when using the PICARD ISRF model. While different attempts to explain the Galactic Center excess have been made, distinguishing the different components by observations remains difficult. While we could show, that the PICARD ISRF model leads to a distinctive increase in the Galactic Center and towards the Galactic Plane, it does not provide a method for observational distinction between the different emission scenarios.

It is necessary to distinguish a strongly-peaked excess in the Galactic Center [30], relating to possible dark matter processes, from an extended excess like reported in [4]. Gaggero et al. 24] find for the later that models with conventional CR physics show at least as good a fit to such an excess as DM related ones 24]. The PICARD ISRF model can account for both excess types, the very localised and an extended component, but has limitations: For the increase in the Galactic Center it is important to mention that the used source distribution using pulsars [54 has its largest uncertainties there. Since IC-related gamma-ray emission is influenced by the local ISRF intensities and the used CR source distribution, reliable predictions in that region still remain difficult. For a larger-scale component, the PICARD ISRF model can account for an increase in the higher $\mathrm{GeV}$ to $\mathrm{TeV}$ regime, well motivated by previous studies like [5], where they account for a possible large-scale component by applying scaling parameters for the ISRF. By introducing further alterations regarding the assumed CR source distribution alongside choosing more adequate Galactic models [23, 33, 35] one might gain sufficient new/underexplored degrees of freedom to provide explanations for the Galactic Center excess problem within conventional CR propagation scenarios without hypothesizing contributions from dark matter annihilation.

Lastly, $\gamma \gamma$-attenuation becomes a relevant competing process at energies above $10 \mathrm{TeV}$, as most recently studied in [42]. Here, we restrict ourself to energies where attenuation did not affect the predictions beyond percent-level. We refrain from 
expanding our energy range presently owing to the transition from the diffusiondominated regime at $\mathrm{GeV}$ energies to the source-dominated regime at $\mathrm{TeV}$ energies and associated uncertainties in 3D-formulation of the $\mathrm{CR}$ source distribution. $\mathrm{Nu}-$ merically efficient 3D propagation models like PICARD now offer the possibility to study 3D source distribution at deca-parsec resolution, and therefore hold promise to deal with adequate propagation physics near CR sources. Recent reports on a CR Pevatron in the Galactic Center by the H.E.S.S. collaboration 3 strongly supports altering or complementing presently used (naive) CR source distributions for application in advanced Galactic CR propagation frameworks. This will be thoroughly investigated in a forthcoming study.

\section{Acknowledgements}

The financial support from the Austrian Science Fund (FWF) project number I1345, in collaboration with the French National Research Agency (ANR), project ID ANR-13-IS05-0001 is acknowledged. The computational results presented have been achieved (in part) using the HPC infrastructure LEO and MACH of the University of Innsbruck. Cristina C Popescu acknowledges support from the Leverhulme Trust Research Project Grant RPG-2013-418. 
[1] Abazajian, K. N., Kaplinghat, M., Oct. 2012. Detection of a gamma-ray source in the Galactic Center consistent with extended emission from dark matter annihilation and concentrated astrophysical emission. Phys. Rev. D86 (8), 083511.

[2] Abdo, A. A., Ackermann, M., Ajello, M., Atwood, W., Axelsson, M., Baldini, L., Ballet, J., Barbiellini, G., Bastieri, D., Battelino, M., et al., 2009. Measurement of the cosmic ray e++ e- spectrum from $20 \mathrm{GeV}$ to $1 \mathrm{TeV}$ with the Fermi Large Area Telescope. Physical Review Letters 102 (18), 181101.

[3] Abramowski, A., Aharonian, F., Benkhali, F. A., Akhperjanian, A. G., Angüner, E. O., Backes, M., Balzer, A., Becherini, Y., Tjus, J. B., et al., Mar. 2016. Acceleration of petaelectronvolt protons in the Galactic Centre. Nature531, 476479 .

[4] Acero, F., Ackermann, M., Ajello, M., Albert, A., Baldini, L., Ballet, J., Barbiellini, G., Bastieri, D., Bellazzini, R., Bissaldi, E., Bloom, E. D., Bonino, R., Bottacini, E., Brandt, T. J., Bregeon, J., Bruel, P., Buehler, R., Buson, S., Caliandro, G. A., Cameron, R. A., Caragiulo, M., Caraveo, P. A., Casandjian, J. M., Cavazzuti, E., Cecchi, C., Charles, E., Chekhtman, A., Chiang, J., Chiaro, G., Ciprini, S., Claus, R., Cohen-Tanugi, J., Conrad, J., Cuoco, A., Cutini, S., D'Ammando, F., de Angelis, A., de Palma, F., Desiante, R., Digel, S. W., Venere, L. D., Drell, P. S., Favuzzi, C., Fegan, S. J., Ferrara, E. C., Focke, W. B., Franckowiak, A., Funk, S., Fusco, P., Gargano, F., Gasparrini, D., Giglietto, N., Giordano, F., Giroletti, M., Glanzman, T., Godfrey, G., Grenier, I. A., Guiriec, S., Hadasch, D., Harding, A. K., Hayashi, K., Hays, E., Hewitt, J. W., Hill, A. B., Horan, D., Hou, X., Jogler, T., Jóhannesson, G., Kamae, T., Kuss, M., Landriu, D., Larsson, S., Latronico, L., Li, J., Li, L., Longo, F., Loparco, F., Lovellette, M. N., Lubrano, P., Maldera, S., Malyshev, D., Manfreda, A., Martin, P., Mayer, M., Mazziotta, M. N., McEnery, J. E., Michelson, P. F., Mirabal, N., Mizuno, T., Monzani, M. E., Morselli, A., Nuss, E., Ohsugi, T., Omodei, N., Orienti, M., Orlando, E., Ormes, J. F., Paneque, D., Pesce-Rollins, M., Piron, F., Pivato, G., Rainò, S., Rando, R., Razzano, M., Razzaque, S., Reimer, A., Reimer, O., Remy, Q., Renault, N., Sánchez-Conde, M., Schaal, M., Schulz, A., Sgrò, C., Siskind, E. J., Spada, F., Spandre, G., Spinelli, P., Strong, A. W., Suson, D. J., Tajima, H., Takahashi, H., Thayer, J. B., Thompson, D. J., Tibaldo, L., Tinivella, M., Torres, D. F., Tosti, G., Troja, E., Vianello, G., Werner, M., Wood, K. S., Wood, M., Zaharijas, G., Zimmer, S., 2016. Development of the Model of Galactic Interstellar Emission for Standard Point-source Analysis of Fermi Large Area Telescope Data. The Astrophysical Journal Supplement Series $223(2), 26$. URL http://stacks . iop.org/0067-0049/223/i=2/a=26

[5] Ackermann, M., Ajello, M., Atwood, W. B., Baldini, L., Ballet, J., Barbiellini, G., Bastieri, D., Bechtol, K., Bellazzini, R., Berenji, B., Blandford, R. D., Bloom, E. D., Bonamente, E., Borgland, A. W., Brandt, T. J., Bregeon, J., Brigida, M., Bruel, P., Buehler, R., Buson, S., Caliandro, G. A., Cameron, R. A., Caraveo, P. A., Cavazzuti, E., Cecchi, C., Charles, E., Chekhtman, A., Chiang, J., Ciprini, S., Claus, R., Cohen-Tanugi, J., Conrad, J., Cutini, S., de Angelis, A., de Palma, F., Dermer, C. D., Digel, S. W., Silva, E. d. C. e., Drell, P. S., Drlica-Wagner, A., Falletti, L., Favuzzi, C., Fegan, S. J., Ferrara, E. C., Focke, W. B., Fortin, P., Fukazawa, Y., Funk, S., Fusco, P., Gaggero, D., Gargano, F., Germani, S., Giglietto, N., Giordano, F., Giroletti, M., Glanzman, T., Godfrey, G., Grove, J. E., Guiriec, S., Gustafsson, M., Hadasch, D., Hanabata, Y., Harding, A. K., 
Hayashida, M., Hays, E., Horan, D., Hou, X., Hughes, R. E., Jóhannesson, G., Johnson, A. S., Johnson, R. P., Kamae, T., Katagiri, H., Kataoka, J., Knödlseder, J., Kuss, M., Lande, J., Latronico, L., Lee, S.-H., Lemoine-Goumard, M., Longo, F., Loparco, F., Lott, B., Lovellette, M. N., Lubrano, P., Mazziotta, M. N., McEnery, J. E., Michelson, P. F., Mitthumsiri, W., Mizuno, T., Monte, C., Monzani, M. E., Morselli, A., Moskalenko, I. V., Murgia, S., Naumann-Godo, M., Norris, J. P., Nuss, E., Ohsugi, T., Okumura, A., Omodei, N., Orlando, E., Ormes, J. F., Paneque, D., Panetta, J. H., Parent, D., Pesce-Rollins, M., Pierbattista, M., Piron, F., Pivato, G., Porter, T. A., Rainò, S., Rando, R., Razzano, M., Razzaque, S., Reimer, A., Reimer, O., Sadrozinski, H. F.-W., Sgrò, C., Siskind, E. J., Spandre, G., Spinelli, P., Strong, A. W., Suson, D. J., Takahashi, H., Tanaka, T., Thayer, J. G., Thayer, J. B., Thompson, D. J., Tibaldo, L., Tinivella, M., Torres, D. F., Tosti, G., Troja, E., Usher, T. L., Vandenbroucke, J., Vasileiou, V., Vianello, G., Vitale, V., Waite, A. P., Wang, P., Winer, B. L., Wood, K. S., Wood, M., Yang, Z., Ziegler, M., Zimmer, S., May 2012. Fermi-LAT Observations of the Diffuse $\gamma$-Ray Emission: Implications for Cosmic Rays and the Interstellar Medium. Astroparticle Journal 750, 3.

[6] Adriani, O., Akaike, Y., Asano, K., Asaoka, Y., Bagliesi, M. G., Bigongiari, G., Binns, W. R., Bonechi, S., Bongi, M., Brogi, P., Buckley, J. H., Cannady, N., Castellini, G., Checchia, C., Cherry, M. L., Collazuol, G., Di Felice, V., Ebisawa, K., Fuke, H., Guzik, T. G., Hams, T., Hareyama, M., Hasebe, N., Hibino, K., Ichimura, M., Ioka, K., Ishizaki, W., Israel, M. H., Javaid, A., Kasahara, K., Kataoka, J., Kataoka, R., Katayose, Y., Kato, C., Kawanaka, N., Kawakubo, Y., Krawczynski, H. S., Krizmanic, J. F., Kuramata, S., Lomtadze, T., Maestro, P., Marrocchesi, P. S., Messineo, A. M., Mitchell, J. W., Miyake, S., Mizutani, K., Moiseev, A. A., Mori, K., Mori, M., Mori, N., Motz, H. M., Munakata, K., Murakami, H., Nakahira, S., Nishimura, J., de Nolfo, G. A., Okuno, S., Ormes, J. F., Ozawa, S., Pacini, L., Palma, F., Papini, P., Penacchioni, A. V., Rauch, B. F., Ricciarini, S. B., Sakai, K., Sakamoto, T., Sasaki, M., Shimizu, Y., Shiomi, A., Sparvoli, R., Spillantini, P., Stolzi, F., Takahashi, I., Takayanagi, M., Takita, M., Tamura, T., Tateyama, N., Terasawa, T., Tomida, H., Torii, S., Tsunesada, Y., Uchihori, Y., Ueno, S., Vannuccini, E., Wefel, J. P., Yamaoka, K., Yanagita, S., Yoshida, A., Yoshida, K., Yuda, T., Nov 2017. Energy spectrum of cosmic-ray electron and positron from 10 gev to 3 tev observed with the calorimetric electron telescope on the international space station. Phys. Rev. Lett. 119, 181101. URL https://link .aps .org/doi/10.1103/PhysRevLett.119.181101

[7] Adriani, O., Barbarino, G. C., Bazilevskaya, G. A., Bellotti, R., Boezio, M., Bogomolov, E. A., Bongi, M., Bonvicini, V., Borisov, S., Bottai, S., Bruno, A., Cafagna, F., Campana, D., Carbone, R., Carlson, P., Casolino, M., Castellini, G., Consiglio, L., De Pascale, M. P., De Santis, C., De Simone, N., Di Felice, V., Galper, A. M., Gillard, W., Grishantseva, L., Jerse, G., Karelin, A. V., Koldashov, S. V., Krutkov, S. Y., Kvashnin, A. N., Leonov, A., Malakhov, V., Malvezzi, V., Marcelli, L., Mayorov, A. G., Menn, W., Mikhailov, V. V., Mocchiutti, E., Monaco, A., Mori, N., Nikonov, N., Osteria, G., Palma, F., Papini, P., Pearce, M., Picozza, P., Pizzolotto, C., Ricci, M., Ricciarini, S. B., Rossetto, L., Sarkar, R., Simon, M., Sparvoli, R., Spillantini, P., Stochaj, S. J., Stockton, J. C., Stozhkov, Y. I., Vacchi, A., Vannuccini, E., Vasilyev, G., Voronov, S. A., Wu, J., Yurkin, Y. T., Zampa, G., Zampa, N., Zverev, V. G., May 2011. CosmicRay Electron Flux Measured by the PAMELA Experiment between 1 and 625 
GeV. Phys. Rev. Lett. 106, 201101.

URL https://link.aps .org/doi/10.1103/PhysRevLett.106.201101

[8] Aharonian, F., Akhperjanian, A., De Almeida, U. B., Bazer-Bachi, A., Becherini, Y., Behera, B., Benbow, W., Bernlöhr, K., Boisson, C., Bochow, A., et al., 2008. Energy spectrum of cosmic-ray electrons at TeV energies. Physical Review Letters 101 (26), 261104.

[9] Ajello, M., Albert, A., Atwood, W. B., Barbiellini, G., Bastieri, D., Bechtol, K., Bellazzini, R., Bissaldi, E., Blandford, R. D., Bloom, E. D., Bonino, R., Bottacini, E., Brandt, T. J., Bregeon, J., Bruel, P., Buehler, R., Buson, S., Caliandro, G. A., Cameron, R. A., Caputo, R., Caragiulo, M., Caraveo, P. A., Cecchi, C., Chekhtman, A., Chiang, J., Chiaro, G., Ciprini, S., Cohen-Tanugi, J., Cominsky, L. R., Conrad, J., Cutini, S., D'Ammando, F., de Angelis, A., de Palma, F., Desiante, R., Venere, L. D., Drell, P. S., Favuzzi, C., Ferrara, E. C., Fusco, P., Gargano, F., Gasparrini, D., Giglietto, N., Giommi, P., Giordano, F., Giroletti, M., Glanzman, T., Godfrey, G., Gomez-Vargas, G. A., Grenier, I. A., Guiriec, S., Gustafsson, M., Harding, A. K., Hewitt, J. W., Hill, A. B., Horan, D., Jogler, T., Jóhannesson, G., Johnson, A. S., Kamae, T., Karwin, C., Knödlseder, J., Kuss, M., Larsson, S., Latronico, L., Li, J., Li, L., Longo, F., Loparco, F., Lovellette, M. N., Lubrano, P., Magill, J., Maldera, S., Malyshev, D., Manfreda, A., Mayer, M., Mazziotta, M. N., Michelson, P. F., Mitthumsiri, W., Mizuno, T., Moiseev, A. A., Monzani, M. E., Morselli, A., Moskalenko, I. V., Murgia, S., Nuss, E., Ohno, M., Ohsugi, T., Omodei, N., Orlando, E., Ormes, J. F., Paneque, D., Pesce-Rollins, M., Piron, F., Pivato, G., Porter, T. A., Rainò, S., Rando, R., Razzano, M., Reimer, A., Reimer, O., Ritz, S., Sánchez-Conde, M., Parkinson, P. M. S., Sgrò, C., Siskind, E. J., Smith, D. A., Spada, F., Spandre, G., Spinelli, P., Suson, D. J., Tajima, H., Takahashi, H., Thayer, J. B., Torres, D. F., Tosti, G., Troja, E., Uchiyama, Y., Vianello, G., Winer, B. L., Wood, K. S., Zaharijas, G., Zimmer, S., 2016. Fermi-LAT Observations of High-Energy Gamma-Ray Emission toward the Galactic Center. The Astrophysical Journal 819 (1), 44. URL http: //stacks . iop.org/0004-637X/819/i=1/a=44

[10] Ambrosi, G., An, Q., Asfandiyarov, R., Azzarello, P., Bernardini, P., Bertucci, B., Cai, M., Chang, J., Chen, D., Chen, H., et al., 2017. Direct detection of a break in the teraelectronvolt cosmic-ray spectrum of electrons and positrons. Nature 552 (7683), 63.

[11] Benyamin, D., Nakar, E., Piran, T., Shaviv, N. J., Jul. 2016. The B/C and Sub-iron/Iron Cosmic Ray Ratios - Further Evidence in Favor of the Spiral-Arm Diffusion Model. ApJ826, 47.

[12] Calore, F., Cholis, I., McCabe, C., Weniger, C., Mar. 2015. A tale of tails: Dark matter interpretations of the Fermi GeV excess in light of background model systematics. Phys. Rev. D91 (6), 063003.

[13] Carlson, E., Profumo, S., Jul. 2014. Cosmic ray protons in the inner Galaxy and the Galactic Center gamma-ray excess. Phys. Rev. D90 (2), 023015.

[14] Cholis, I., Evoli, C., Calore, F., Linden, T., Weniger, C., Hooper, D., Dec. 2015. The Galactic Center GeV excess from a series of leptonic cosmic-ray outbursts. J. Cosmology Astropart. Phys.12, 005. 
[15] Davies, L. J. M., Driver, S. P., Robotham, A. S. G., Grootes, M. W., Popescu, C. C., Tuffs, R. J., Hopkins, A., Alpaslan, M., Andrews, S. K., Bland-Hawthorn, J., Bremer, M. N., Brough, S., Brown, M. J. I., Cluver, M. E., Croom, S., da Cunha, E., Dunne, L., Lara-López, M. A., Liske, J., Loveday, J., Moffett, A. J., Owers, M., Phillipps, S., Sansom, A. E., Taylor, E. N., Michalowski, M. J., Ibar, E., Smith, M., Bourne, N., Sep. 2016. GAMA/H-ATLAS: a meta-analysis of SFR indicators - comprehensive measures of the SFR-M $\mathrm{M}_{*}$ relation and cosmic star formation history at $\mathrm{z}<0.4$. MNRAS461, 458-485.

[16] Daylan, T., Finkbeiner, D. P., Hooper, D., Linden, T., Portillo, S. K. N., Rodd, N. L., Slatyer, T. R., Jun. 2016. The characterization of the gamma-ray signal from the central Milky Way: A case for annihilating dark matter. Physics of the Dark Universe 12, 1-23.

[17] Driver, S. P., Popescu, C. C., Tuffs, R. J., Graham, A. W., Liske, J., Baldry, I., May 2008. The Energy Output of the Universe from 0.1 to $1000 \mu \mathrm{m}$. ApJ678, L101.

[18] Driver, S. P., Popescu, C. C., Tuffs, R. J., Liske, J., Graham, A. W., Allen, P. D., de Propris, R., Aug. 2007. The Millennium Galaxy Catalogue: the B-band attenuation of bulge and disc light and the implied cosmic dust and stellar mass densities. MNRAS379, 1022-1036.

[19] Driver, S. P., Robotham, A. S. G., Kelvin, L., Alpaslan, M., Baldry, I. K., Bamford, S. P., Brough, S., Brown, M., Hopkins, A. M., Liske, J., Loveday, J., Norberg, P., Peacock, J. A., Andrae, E., Bland-Hawthorn, J., Bourne, N., Cameron, E., Colless, M., Conselice, C. J., Croom, S. M., Dunne, L., Frenk, C. S., Graham, A. W., Gunawardhana, M., Hill, D. T., Jones, D. H., Kuijken, K., Madore, B., Nichol, R. C., Parkinson, H. R., Pimbblet, K. A., Phillipps, S., Popescu, C. C., Prescott, M., Seibert, M., Sharp, R. G., Sutherland, W. J., Taylor, E. N., Thomas, D., Tuffs, R. J., van Kampen, E., Wijesinghe, D., Wilkins, S., Dec. 2012. Galaxy And Mass Assembly (GAMA): the $0.013<\mathrm{z}<0.1$ cosmic spectral energy distribution from $0.1 \mu \mathrm{m}$ to $1 \mathrm{~mm}$. MNRAS427, 3244-3264.

[20] Evoli, C., Gaggero, D., Grasso, D., Maccione, L., Oct. 2008. Cosmic ray nuclei, antiprotons and gamma rays in the galaxy: a new diffusion model. J. Cosmology Astropart. Phys.10, 018.

[21] Gaggero, D., Grasso, D., Marinelli, A., Urbano, A., Valli, M., 2016. Gammaray and neutrino diffuse emissions of the Galaxy above the TeV. In: Journal of Physics: Conference Series. Vol. 718. IOP Publishing, p. 052018.

[22] Gaggero, D., Maccione, L., Di Bernardo, G., Evoli, C., Grasso, D., Jul. 2013. Three-Dimensional Model of Cosmic-Ray Lepton Propagation Reproduces Data from the Alpha Magnetic Spectrometer on the International Space Station. Physical Review Letters 111 (2), 021102.

[23] Gaggero, D., Maccione, L., Di Bernardo, G., Evoli, C., Grasso, D., Jun. 2013. Three dimensional modeling of CR propagation. ArXiv e-prints.

[24] Gaggero, D., Taoso, M., Urbano, A., Valli, M., Ullio, P., 2015. Towards a realistic astrophysical interpretation of the gamma-ray Galactic center excess. Journal of Cosmology and Astroparticle Physics 2015 (12), 056.

URL http://stacks . iop.org/1475-7516/2015/i=12/a=056 
[25] Goodenough, L., Hooper, D., Oct. 2009. Possible Evidence For Dark Matter Annihilation In The Inner Milky Way From The Fermi Gamma Ray Space Telescope. ArXiv e-prints.

[26] Gordon, C., Macías, O., Feb 2014. Erratum: Dark matter and pulsar model constraints from Galactic Center Fermi-LAT gamma-ray observations [Phys. Rev. D 88, 083521 (2013)]. Phys. Rev. D 89, 049901.

URL https://link.aps .org/doi/10.1103/PhysRevD.89.049901

[27] Grootes, M. W., Tuffs, R. J., Popescu, C. C., Norberg, P., Robotham, A. S. G., Liske, J., Andrae, E., Baldry, I. K., Gunawardhana, M., Kelvin, L. S., Madore, B. F., Seibert, M., Taylor, E. N., Alpaslan, M., Brown, M. J. I., Cluver, M. E., Driver, S. P., Bland-Hawthorn, J., Holwerda, B. W., Hopkins, A. M., LopezSanchez, A. R., Loveday, J., Rushton, M., Mar. 2017. Galaxy And Mass Assembly (GAMA): Gas Fueling of Spiral Galaxies in the Local Universe. I. The Effect of the Group Environment on Star Formation in Spiral Galaxies. AJ153, 111.

[28] Grootes, M. W., Tuffs, R. J., Popescu, C. C., Pastrav, B., Andrae, E., Gunawardhana, M., Kelvin, L. S., Liske, J., Seibert, M., Taylor, E. N., Graham, A. W., Baes, M., Baldry, I. K., Bourne, N., Brough, S., Cooray, A., Dariush, A., De Zotti, G., Driver, S. P., Dunne, L., Gomez, H., Hopkins, A. M., Hopwood, R., Jarvis, M., Loveday, J., Maddox, S., Madore, B. F., Michałowski, M. J., Norberg, P., Parkinson, H. R., Prescott, M., Robotham, A. S. G., Smith, D. J. B., Thomas, D., Valiante, E., Mar. 2013. GAMA/H-ATLAS: The Dust Opacity-Stellar Mass Surface Density Relation for Spiral Galaxies. ApJ766, 59.

[29] Hooper, D., Goodenough, L., 2011. Dark matter annihilation in the Galactic Center as seen by the Fermi Gamma Ray Space Telescope. Physics Letters B $697(5), 412-428$.

URL

http://wwW.sciencedirect.com/science/article/pii/ S0370269311001742

[30] Hooper, D., Linden, T., Dec 2011. Origin of the gamma rays from the Galactic Center. Phys. Rev. D 84, 123005.

URL https://link .aps .org/doi/10.1103/PhysRevD .84.123005

[31] Hooper, D., Slatyer, T. R., 2013. Two emission mechanisms in the Fermi Bubbles: A possible signal of annihilating dark matter. Physics of the Dark Universe 2 (3), 118-138.

URL http://www.sciencedirect.com/science/article/pii/ S2212686413000216

[32] Jóhannesson, G., Moskalenko, I. V., Porter, T. A., 2017. Interstellar gas in 3D, implications for CR propagation and gamma-ray emission. URL https://pos.sissa.it/301/871

[33] Kissmann, R., Werner, M., Reimer, O., Strong, A. W., Oct. 2015. Propagation in 3D spiral-arm cosmic-ray source distribution models and secondary particle production using PICARD. Astroparticle Physics 70, 39-53.

[34] Linden, T., Lovegrove, E., Profumo, S., 2012. The morphology of hadronic emission models for the gamma-ray source at the galactic center. The Astrophysical Journal 753 (1), 41.

URL http://stacks .iop.org/0004-637X/753/i=1/a=41 
[35] Moskalenko, I. V., Jóhannesson, G., Orlando, E., Porter, T. A., Strong, A. W., 2017. GALPROP Code for Galactic Cosmic Ray Propagation and Associated Photon Emissions.

URL https://pos.sissa.it/301/279

[36] Moskalenko, I. V., Porter, T. A., Strong, A. W., Apr. 2006. Attenuation of Very High Energy Gamma Rays by the Milky Way Interstellar Radiation Field. ApJ640, L155-L158.

[37] Pastrav, B. A., Popescu, C. C., Tuffs, R. J., Sansom, A. E., May 2013. The effects of dust on the derived photometric parameters of disks and bulges in spiral galaxies. A\&A553, A80.

[38] Pastrav, B. A., Popescu, C. C., Tuffs, R. J., Sansom, A. E., Sep. 2013. The effects of dust on the photometric parameters of decomposed disks and bulges. A\&A557, A137.

[39] Popescu, C. C., Misiriotis, A., Kylafis, N. D., Tuffs, R. J., Fischera, J., Oct. 2000. Modelling the spectral energy distribution of galaxies. I. Radiation fields and grain heating in the edge-on spiral NGC 891. A\&A362, 138-150.

[40] Popescu, C. C., Tuffs, R. J., Dopita, M. A., Fischera, J., Kylafis, N. D., Madore, B. F., Mar. 2011. Modelling the spectral energy distribution of galaxies. V. The dust and PAH emission SEDs of disk galaxies. A\&A527, A109.

[41] Popescu, C. C., Tuffs, R. J., Kylafis, N. D., Madore, B. F., Jan. 2004. Far Infrared mapping of NGC 891. A\&A414, 45-52.

[42] Popescu, C. C., Yang, R., Tuffs, R. J., Natale, G., Rushton, M., Aharonian, F., Sep. 2017. A radiation transfer model for the Milky Way: I. Radiation fields and application to high-energy astrophysics. MNRAS470, 2539-2558.

[43] Porter, T. A., Jóhannesson, G., Moskalenko, I. V., 2017. High-Energy $\gamma$-rays from the Milky Way: Three-Dimensional Spatial Models for the Cosmic-Ray and Radiation Field Densities. Proceedings of Science 301 (35th International Cosmic Ray Conference).

URL https://pos.sissa.it/301/736

[44] Porter, T. A., Jóhannesson, G., Moskalenko, I. V., 2017. High-energy Gamma Rays from the Milky Way: Three-dimensional Spatial Models for the CosmicRay and Radiation Field Densities in the Interstellar Medium. The Astrophysical Journal 846 (1), 67. URL http://stacks .iop.org/0004-637X/846/i=1/a=67

[45] Porter, T. A., Moskalenko, I. V., Strong, A. W., Sep. 2006. Inverse Compton Emission from Galactic Supernova Remnants: Effect of the Interstellar Radiation Field. ApJ648, L29-L32.

[46] Porter, T. A., Moskalenko, I. V., Strong, A. W., Orlando, E., Bouchet, L., Jul. 2008. Inverse Compton Origin of the Hard X-Ray and Soft Gamma-Ray Emission from the Galactic Ridge. The Astrophysical Journal 682, 400-407.

[47] R. Kissmann, O. Reimer, A. Strong, M. Werner, June 2014. Picard: A new Code for The Galactic Cosmic Ray Propagation Problem. Proceedings of Science. 
[48] Rybicki, G. B., Lightman, A. P., May 1985. Radiative Processes in Astrophysics. John Wiley \& Sons.

[49] Strong, A. W., Moskalenko, I. V., Dec. 1998. Propagation of Cosmic-Ray Nucleons in the Galaxy. ApJ509, 212-228.

[50] Strong, A. W., Moskalenko, I. V., Ptuskin, V. S., 2007. Cosmic-Ray Propagation and Interactions in the Galaxy. Annual Review of Nuclear and Particle Science 57 (1), 285-327.

URL https://doi.org/10.1146/annurev.nucl.57.090506.123011

[51] Vulcani, B., Bamford, S. P., Häußler, B., Vika, M., Rojas, A., Agius, N. K., Baldry, I., Bauer, A. E., Brown, M. J. I., Driver, S., Graham, A. W., Kelvin, L. S., Liske, J., Loveday, J., Popescu, C. C., Robotham, A. S. G., Tuffs, R. J., Jun. 2014. Galaxy And Mass Assembly (GAMA): the wavelength-dependent sizes and profiles of galaxies revealed by MegaMorph. MNRAS441, 1340-1362.

[52] Weingartner, J. C., Draine, B. T., Feb. 2001. Dust Grain-Size Distributions and Extinction in the Milky Way, Large Magellanic Cloud, and Small Magellanic Cloud. ApJ548, 296-309.

[53] Werner, M., Kissmann, R., Strong, A. W., Reimer, O., Apr. 2015. Spiral arms as cosmic ray source distributions. Astroparticle Physics 64, 18-33.

[54] Yusifov, I., Küçük, I., Aug. 2004. Revisiting the radial distribution of pulsars in the Galaxy. A\&A422, 545-553. 